\title{
Inversion symmetric non-Hermitian Chern insulator
}

\author{
H. C. Wu, L. Jin $\odot,^{*}$ and Z. Song \\ School of Physics, Nankai University, Tianjin 300071, China
}

(Received 27 May 2019; published 8 October 2019)

\begin{abstract}
We propose a two-dimensional non-Hermitian Chern insulator with inversion symmetry, which is anisotropic and has staggered gain and loss in both $x$ and $y$ directions. In this system, conventional bulk-boundary correspondence holds. The Chern number is a topological invariant that accurately predicts the topological phase transition and the existence of helical edge states in the topologically nontrivial gapped phase. In the gapless phase, the band touching points are isolated and protected by the symmetry. The degenerate points alter the system topology, and the exceptional points can destroy the existence of helical edge states. Topologically protected helical edge states exist in the gapless phase for the system under open boundary condition in one direction, which are predicted by the winding number associated with the vector field of average values of Pauli matrices. The winding number also identifies the detaching points between the edge states and the bulk states in the energy bands. The non-Hermiticity also supports a topological phase with zero Chern number, where a pair of in-gap helical edge states exists. Our findings provide insights into the symmetry protected non-Hermitian topological insulators.
\end{abstract}

DOI: 10.1103/PhysRevB.100.155117

\section{INTRODUCTION}

The topological phase of matter in condensed matter physics has been attracting considerable research interest and has been widely explored [1-7]. Open systems ubiquitously exist in physics [8-10], particularly, the optical and photonic systems; these are mostly non-Hermitian because they interact with the environment [11-16]. Currently, topological systems extend into the non-Hermitian region [17-44], and the nontrivial topological properties are studied in one-dimensional (1D), two-dimensional (2D), and threedimensional (3D) systems, including the Su-SchriefferHeeger (SSH) model [45-49], Aubry-André-Harper (AAH) model [50-55], Rice-Mele (RM) model [56,57], Chern insulator [58-62], and Weyl semimetal [63,64].

Edge states in the parity-time $(\mathcal{P} \mathcal{T})$-symmetric systems are considered absent because the edge state with its probability localized at one system boundary is not $\mathcal{P} \mathcal{T}$ invariant [21]. However, $\mathcal{P} \mathcal{T}$-symmetric interface states can localize at the interface or domain wall of two configurations with different topologies [65,66]; a zero mode can appear at the interface of lattices in the same topological phase but with different nonHermitian phases [67]. Both the types of interface states are topologically protected and robust to disorders. Topological edge states lasing in $1 \mathrm{D}$ [68-71] and 2D [72-75] have been reported recently; robust single-mode lasing prevails due to the topological protection. Non-Hermitian topological sys-

\footnotetext{
*jinliang@nankai.edu.cn
}

Published by the American Physical Society under the terms of the Creative Commons Attribution 4.0 International license. Further distribution of this work must maintain attribution to the author(s) and the published article's title, journal citation, and DOI. tems with $\mathcal{P} \mathcal{T}$ symmetry are of primary focus [76-82]. Other symmetries, such as chiral-time $(\mathcal{C} \mathcal{T})$ and charge-parity $(\mathcal{C P})$ symmetries, have been investigated $[24,41,48,83,84]$. The topological aspects of non-Hermitian systems, including edge modes [84,85], topological invariant [63,86-92], band theory [92-95], topological pumping [56,59,96-98], classification [99-105], high-order non-Hermitian topological systems [106-111], semimetals [112-117], and symmetry-protected topological phases and localized states have been investigated [90,118-121]. The exceptional points (EPs) of non-Hermitian systems are connected by Fermi arcs [122-125] or form EP rings and surfaces [64,126-128].

Notably, the bulk-boundary correspondence fails in some non-Hermitian topological systems $[57,61,99,129-142]$. The spectrum under the periodical boundary condition (PBC) significantly differs from that under the open boundary condition $(\mathrm{OBC})$, and the eigenstates under $\mathrm{OBC}$ are all localized at the system boundary (the non-Hermitian skin effect) [133]. The topological invariant can be constructed either from the biorthogonal norm [57], the non-Bloch bulk [61,133], or the singular-value decomposition of the Hamiltonian [140]. The reason for the breakdown of bulk-boundary correspondence is that an asymmetric coupling induces an imaginary AharonovBohm effect $[134,135]$; the validity of the bulk-boundary correspondence can be maintained by chiral-inversion symmetry [134]. The boundary modes in non-Hermitian systems have been discussed on the basis of the transfer matrix method [143] and the Green's function method [85,144,145]. The interplay between non-Hermiticity and non-Abelian gauge potential has been discussed [146]. In contrast to the topological phase transition, the invalidity of bulk-boundary correspondence, and the non-Hermitian skin effect, non-Hermiticity may not alter the topological phase transition and system topology [147]. A graphical approach has been proposed to 
visualize the topological phases in non-Hermitian systems [148].

Different from the non-Hermitian Chern insulators in Refs. [58-62], an inversion symmetric 2D non-Hermitian Chern insulator is proposed and the validity of conventional bulk-boundary correspondence is predicted in the end of Ref. [134]. However, more physical aspects including the topological invariant and the edge state are not discussed. In this study, the inversion symmetric non-Hermitian Chern insulator is investigated in detail; in particular, the topological properties of the gapless phase as well as the technical aspect of topological characterization are systematically analyzed. Notably, the gain and loss are alternately added in the $x$ and $y$ directions in the inversion symmetric non-Hermitian Chern insulator. The non-Hermitian Aharonov-Bohm effect and skin effect are prevented by the inversion symmetry, and the conventional bulk-boundary correspondence is valid. The Chern number constructed from the system bulk is a topological invariant used to predict the topological properties of the Chern insulator. Different from the anomalous edge states that are localized in a single unit cell [129], and those that cannot be predicted by the bulk topology [60], a pair of helical edge states appear in the topologically nontrivial phase of the inversion symmetric Chern insulator under OBC. The gapless phase has band touching points in the Brillouin zone (BZ). The locations of the band touching degenerate points (DPs) are fixed and they do not change into pairs of EPs [124]. The band touching EPs are isolated and topologically protected, moving in the $\mathrm{BZ}$ and merging when they meet in pairs. Band touching varies the system topology and the existence of helical edge states. Moreover, non-Hermiticity creates a pair of topologically protected in-gap helical edge states in a novel phase with zero Chern number.

The remainder of the paper is organized as follows. In Sec. II, we introduce the inversion symmetric 2D nonHermitian Chern insulator. In Sec. III, we discuss the energy bands, the phase diagram, and the topological characterization of the bulk Hamiltonian. In Sec. IV, we demonstrate the energy spectrum and the helical edge states in different topological phases of the edge Hamiltonian, and we verify the validity of conventional bulk-boundary correspondence. In Sec. V, we present the connection between the 2D nonHermitian Chern insulator and other quasi-1D non-Hermitian topological systems with asymmetric couplings. In addition, we discuss possible experimental realization. Finally, in Sec. VI, we summarize the main findings.

\section{INVERSION SYMMETRIC NON-HERMITIAN CHERN INSULATOR}

We investigate a non-Hermitian 2D topological system with inversion symmetry. The schematic of the lattice of the non-Hermitian 2D topological system is presented in Fig. 1. The lattice in the Hermitian case is a Chern insulator, constituted by vertical Creutz ladders [149]. The Creutz ladders are horizontally coupled with strength $t$. The intraladder rung represents a coupling with strength $m$. Couplings of strength $m$ and $t$ are alternated along the $x$ direction. Along the $y$ direction, the $t / 2$ coupling has an additional $\pm \pi / 2$ Peierls phase factor in the front, which results in magnetic flux $\pi$

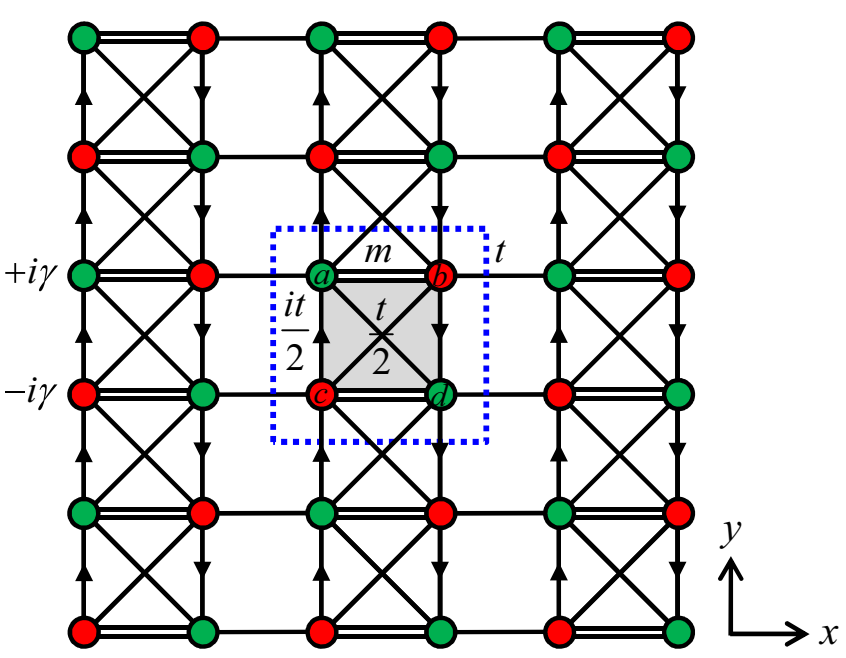

FIG. 1. Schematic of the inversion symmetric 2D non-Hermitian Chern insulator. The green (red) solid circle indicates the site with gain (loss). The Peierls phase factor is $e^{i \pi / 2}$ in the nonreciprocal coupling it $/ 2$ in the vertical direction. The Peierls phase leads to a flux of $\pi$ in each plaquette.

in each plaquette, indicated by the shaded square in Fig. 1. The off-diagonal coupling strength in the plaquette is $t / 2$, which is equivalent to spin-orbital coupling [150]. The lattice Hamiltonian in the real space is $H=H_{0}+H_{1}$, consisting of the nearest-neighbor coupling term

$$
\begin{aligned}
H_{0}= & \sum_{i, j}\left[m\left(a_{i, j}^{\dagger} b_{i, j}+c_{i, j}^{\dagger} d_{i, j}\right)+t\left(a_{i+1, j}^{\dagger} b_{i, j}+c_{i+1, j}^{\dagger} d_{i, j}\right)\right. \\
& \left.+\frac{i t}{2}\left(a_{i, j}^{\dagger} c_{i, j}-a_{i, j-1}^{\dagger} c_{i, j}-b_{i, j}^{\dagger} d_{i, j}+b_{i, j-1}^{\dagger} d_{i, j}\right)\right]+ \text { H.c. }
\end{aligned}
$$

and the off-diagonal coupling term

$$
H_{1}=\sum_{i, j} \frac{t}{2}\left(a_{i, j}^{\dagger} d_{i, j}+a_{i, j-1}^{\dagger} d_{i, j}+b_{i, j}^{\dagger} c_{i, j}+b_{i, j-1}^{\dagger} c_{i, j}\right)+\text { H.c., }
$$

where the operators $a_{i, j}^{\dagger}, b_{i, j}^{\dagger}, c_{i, j}^{\dagger}, d_{i, j}^{\dagger}$ are creation operators for the four sublattices in the unit cell $(i, j)$. The unit cell is indicated in Fig. 1 by the blue square with dashed lines. The system exhibits inversion symmetry, and the real-space Hamiltonian $H$ is invariant under a rotation of $\pi$ with respect to the lattice center. The inversion symmetry in 1D systems is the reflection symmetry. The topological classification of non-Hermitian systems with reflection symmetry and the rich topological phases are studied [103].

We consider generalization of the non-Hermitian system holding inversion symmetry. The gain and loss with rates $\gamma$ are alternately introduced in both $x$ and $y$ directions of $H$; the non-Hermitian Hamiltonian is $\mathcal{H}=H+H_{\gamma}$ and consists of staggered gain and loss

$$
H_{\gamma}=i \gamma \sum_{i, j}\left(a_{i, j}^{\dagger} a_{i, j}-b_{i, j}^{\dagger} b_{i, j}-c_{i, j}^{\dagger} c_{i, j}+d_{i, j}^{\dagger} d_{i, j}\right) .
$$


Notably, the generalized non-Hermitian Chern insulator $\mathcal{H}$ holds the inversion symmetry and differs from other nonHermitian Chern insulators without inversion symmetry in Refs. $[60,61]$. The inversion symmetry ensures the presence of a zero imaginary magnetic flux [134]. Thus the non-Hermitian skin effect is absent and the conventional bulk-boundary correspondence holds in the inversion symmetric non-Hermitian Chern insulator. The topological properties of the system can be retrieved from its bulk topology. In the following sections, the energy bands, phase diagram, topological characterization, and edge states are elucidated.

\section{PHASE DIAGRAM AND TOPOLOGICAL CHARACTERIZATION}

Bloch Hamiltonian. We apply the Fourier transformation $\rho_{k_{x}, k_{y}}=N^{-1 / 2} \sum_{l, s} e^{-i k_{x} l} e^{-i k_{y} s} \rho_{l, s}$ to the sublattices $\rho=$ $a, b, c, d$; the Hamiltonian of the non-Hermitian system in the real space is rewritten in the momentum space, $\mathcal{H}=$ $\sum_{\mathbf{k}} \mathcal{H}(\mathbf{k})$. The Bloch Hamiltonian of the non-Hermitian system in the basis $\left\{a_{k_{x}, k_{y}}^{\dagger}|\mathrm{vac}\rangle, b_{k_{x}, k_{y}}^{\dagger}|\mathrm{vac}\rangle, c_{k_{x}, k_{y}}^{\dagger}|\mathrm{vac}\rangle, d_{k_{x}, k_{y}}^{\dagger}|\mathrm{vac}\rangle\right\}$ reads

$$
\mathcal{H}(\mathbf{k})=\left(\begin{array}{cccc}
i \gamma & m+t e^{-i k_{x}} & \Lambda_{-}\left(k_{y}\right) & \Lambda_{+}\left(k_{y}\right) \\
m+t e^{i k_{x}} & -i \gamma & \Lambda_{+}\left(k_{y}\right) & -\Lambda_{-}\left(k_{y}\right) \\
\Lambda_{-}^{*}\left(k_{y}\right) & \Lambda_{+}^{*}\left(k_{y}\right) & -i \gamma & m+t e^{-i k_{x}} \\
\Lambda_{+}^{*}\left(k_{y}\right) & -\Lambda_{-}^{*}\left(k_{y}\right) & m+t e^{i k_{x}} & i \gamma
\end{array}\right),
$$

where we have $\Lambda_{+}\left(k_{y}\right)=t\left(1+e^{i k_{y}}\right) / 2$ and $\Lambda_{-}\left(k_{y}\right)=i t(1-$ $\left.e^{i k_{y}}\right) / 2$. The Bloch Hamiltonian has the inversion symmetry $\mathcal{P H}(\mathbf{k}) \mathcal{P}^{-1}=\mathcal{H}(-\mathbf{k})$ with $\mathcal{P}=\sigma_{x} \otimes \sigma_{x}$, and the particlehole (charge-conjugation) symmetry $\mathcal{C H}(\mathbf{k}) \mathcal{C}^{-1}=-\mathcal{H}(-\mathbf{k})$ with $\mathcal{C}=\left(\sigma_{0} \otimes \sigma_{z}\right) \mathcal{K}$, where $\mathcal{K}$ is the complex conjugation operation; $\sigma_{x}, \sigma_{y}$, and $\sigma_{z}$ are the Pauli matrices; and $\sigma_{0}$ is a $2 \times 2$ identity matrix.

The particle-hole symmetry ensures the spectrum of $\mathcal{H}(\mathbf{k})$ to be symmetric about zero energy, with the energy bands given by

$$
E_{ \pm, \pm}(\mathbf{k})= \pm \sqrt{h_{x, \pm}^{2}+h_{y}^{2}+h_{z}^{2}}
$$

where $\mu=m+t \cos k_{x}+\gamma, v=m+t \cos k_{x}-\gamma$, and $h_{x, \pm}=\sqrt{\mu \nu} \pm t \cos \left(k_{y} / 2\right), h_{y}=t \sin \left(k_{y} / 2\right)$, and $h_{z}=t \sin k_{x}$. For $\mu \nu<0, \sqrt{\mu \nu}$ is imaginary and the energy spectrum is complex. Highly defective exceptional lines (HDELs) appear at $\mu \nu=0$, that is, when $t \cos k_{x}=-m \pm \gamma$. The HDELs are fully constituted by EPs, and the energy levels are two-state coalesced in pairs at energy $\pm \sqrt{t^{2}+h_{z}^{2}}$. The energies are indicated by the black lines in Fig. 2. Notably, the HDELs are EP lines across the BZ. The upper and lower bands shrink into two levels with opposite energies. In the $\mathrm{BZ}$, zero to four HDELs symmetrically appear about $k_{x}=$ 0 at different system parameters. In addition, the band gap closes at zero energy, where the band touching points can be either DPs $\left[h_{x,-(+)}=h_{y}=h_{z}=0\right]$ or EPs. The inversion symmetry leads to an inversion symmetric distribution of the band touching points in the $\mathrm{BZ}$ and the band touching points always appear in pairs of $\left(k_{x}, k_{y}\right)$ and $\left(-k_{x},-k_{y}\right)$. Figure 2 depicts the absolute value of the energy spectrum $\left|E_{+, \pm}(\mathbf{k})\right|$. From Fig. 2(a) to Fig. 2(f), the non-Hermiticity
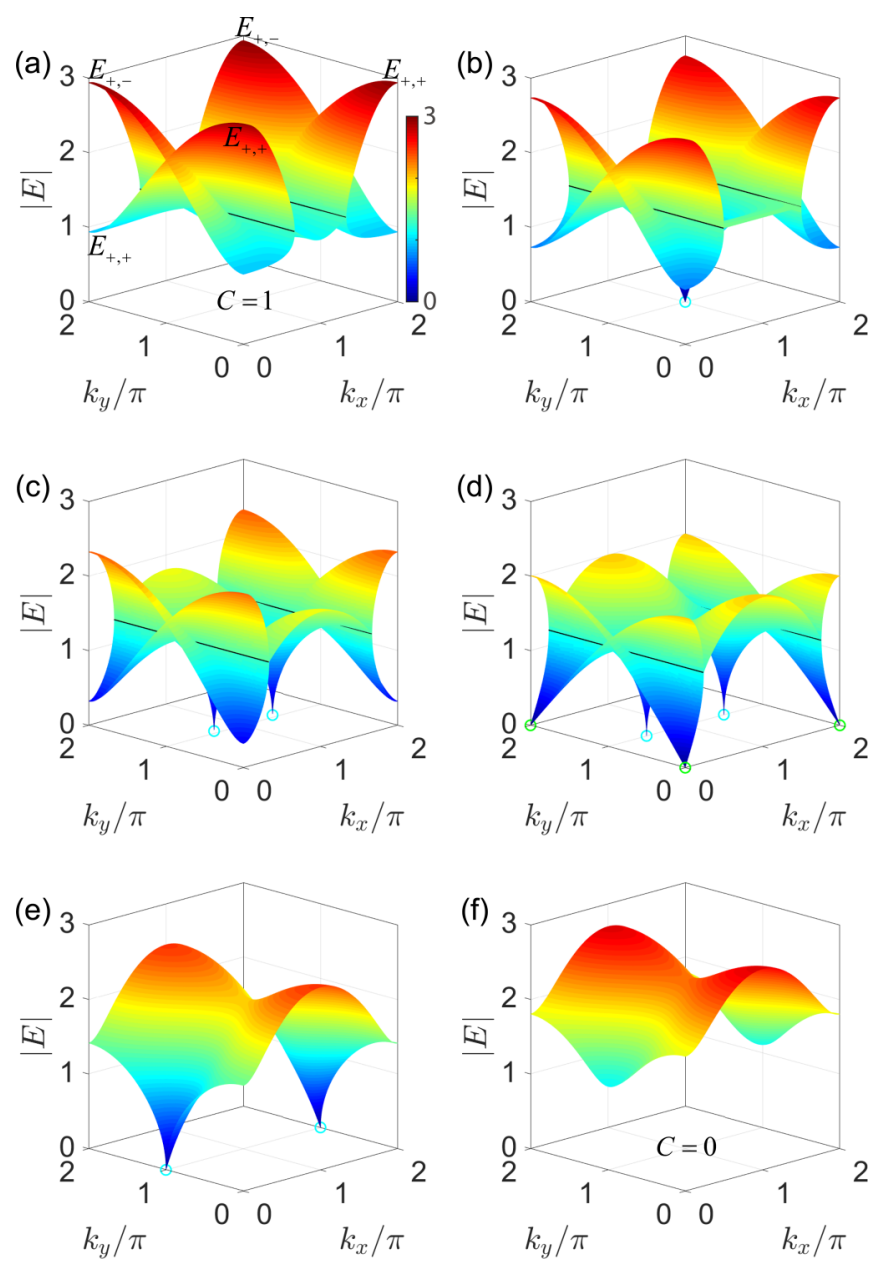

FIG. 2. Energy bands of the non-Hermitian Chern insulator $\mathcal{H}(\mathbf{k})$ at $m=t$ for various $\gamma$. (a) $\gamma=1 / 2$, (b) 1 , (c) $3 / 2$, (d) $\sqrt{3}$, (e) $\sqrt{5}$, and (f) $5 / 2$. The parameters are indicated by the hollow blue circles in the phase diagram shown in Fig. 3. The absolute value of the upper band energy $\left|E_{+, \pm}(\mathbf{k})\right|$ is depicted. The EPs (DPs) in the gapless phase are marked by the cyan (green) circles. The black lines indicate the energy at the HDELs. All color bars are identical to the one shown in (a).

increases from $\gamma / t=1 / 2$ to $\gamma / t=5 / 2$. The energy bands satisfy $E_{+(-),+}\left(k_{x}, k_{y}\right)=E_{+(-),-}\left(k_{x}, k_{y}+2 \pi\right)$. The two upper (lower) energy bands of the inversion symmetric nonHermitian Chern insulator constitute an entire band without intersection if one of the two bands $E_{+, \pm}(\mathbf{k})\left[E_{-, \pm}(\mathbf{k})\right]$ shifted by $2 \pi$ along the $k_{y}$ direction in the BZ. The constituted band is the upper (lower) band of Eq. (10) by substituting $k_{y}$ with $k_{y} / 2$; the corresponding energy bands are depicted in the Appendix A.

Phase diagram. To analyze the phase diagram of the system in detail, we consider $t$ as the unit. The phase diagram of the non-Hermitian Chern insulator is depicted in Fig. 3 as a function of system parameters $m$ and $\gamma$. In the Hermitian situation with $\gamma=0$, the energy bands are gapped for $m / t \neq 0, \pm 2$ and the Chern number is $C=1(C=-1)$ for $0<m / t<2(-2<m / t<0)$. In case $|m / t|>2$, the Chern number is zero. In the non-Hermitian situation with $\gamma \neq 0$, instead of splitting into pairs of EPs [124], the band touching 


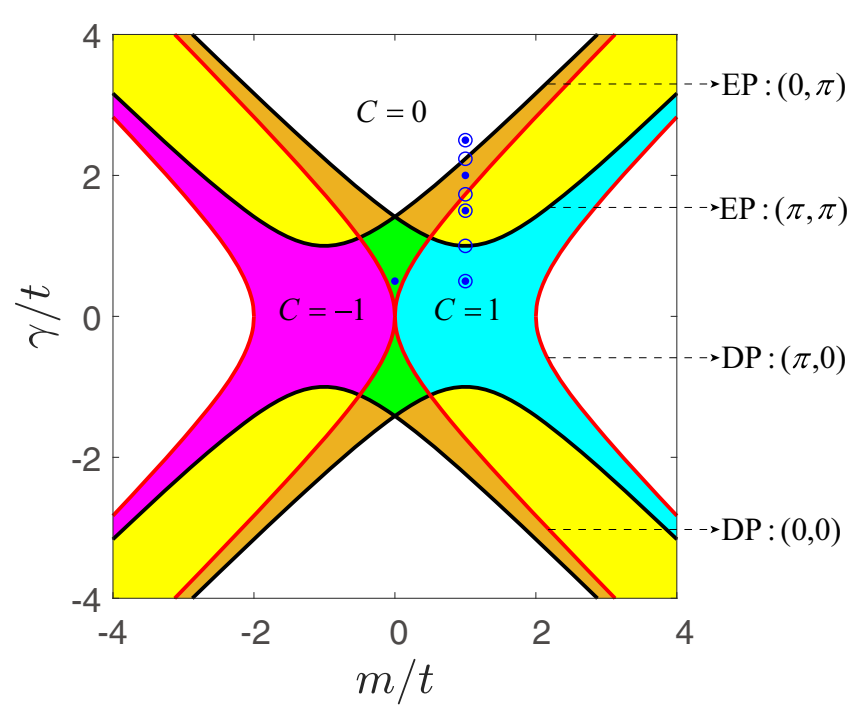

FIG. 3. Phase diagram in the $m-\gamma$ parameter space. The yellow and orange regions indicate the gapless phase. In the phase with separable bands, the Chern number is nonzero in the magenta and cyan regions; the Chern number is zero in the white and green regions. The black (red) curves indicate the gapless phase with EPs (DPs), where the Chern number is not available and winding number [Eq. (12)] is employed as a topological invariant. For the topological phase with nonzero Chern number, the helical edge states exist for the system under OBC in the $x$ direction or in the $y$ direction. In the gapless phase, the helical edge states exist for the system under OBC only in one direction. In-gap helical edge states exist in the green region. The hollow blue circles (solid blue dots) indicate the parameters chosen in Fig. 2 (Fig. 7).

DPs appear at fixed positions in the BZ, with their appearance distinguishing the topologically nontrivial and trivial phases. In the gapless phase, the band touching DPs occur at $\sqrt{\mu \nu}-t \cos \left(k_{y} / 2\right)=h_{y}=h_{z}=0$, which depends on nonHermiticity given by

$$
\gamma^{2}=(m \pm t)^{2}-t^{2}
$$

This is indicated by the red curves in the phase diagram in Fig. 3. The DP locations are fixed in the BZ and at $\left(k_{x}, k_{y}\right)=$ $(0,0)$ for $\gamma^{2}=(m+t)^{2}-t^{2}$ and at $\left(k_{x}, k_{y}\right)=(\pi, 0)$ for $\gamma^{2}=(m-t)^{2}-t^{2}$.

The yellow and orange regions in Fig. 3 represent the gapless phase with two symmetry protected EPs. The EPs possess distinct topology from that of DPs due to the bifurcation linking the Riemann surface [151-164]. The band touching points are EPs with fractional charge $\pm 1 / 2$ according to the definition of non-Hermitian winding number $\mathcal{W}_{\mathrm{EP}}=$ $-(2 \pi)^{-1} \oint_{\Gamma} \nabla_{\mathbf{k}} \arg \left[E_{+}(\mathbf{k})-E_{-}(\mathbf{k})\right] d \mathbf{k}[92]$, where $\Gamma$ is a closed loop in the momentum space. In these gapless regions, the symmetry protected EPs move in the BZ as system parameters, and merge when they meet in the BZ due to topological phase transition; then, the number of EPs in the BZ reduces to one and the topological features of EP change. As $\gamma$ increases, the separate upper and lower bands become closer and may touch at $|\gamma / t|>1$. The band touching EPs appear in the regions

$$
(|m|-t)^{2}+t^{2} \leqslant \gamma^{2} \leqslant(|m|+t)^{2}+t^{2} .
$$

At $m=0$, we have $\gamma^{2}=2 t^{2}$; the EPs form EP lines along $k_{y}=\pi$. Otherwise at $m \neq 0$, two EPs appear at $\left(k_{x}, k_{y}\right)=$ $( \pm \theta, \pi)$ when the system is within the gapless regions, where $\theta$ is obtained from $\cos \theta=\left(\gamma^{2}-m^{2}-2 t^{2}\right) /(2 m t)$. The EPs in the BZ appear and move along $k_{y}=\pi$ as system parameters, and symmetrically distribute about $k_{x}=0$ with different chiralities [151,152]. At the boundary of the gapless phase represented by the black curves in the phase diagram Fig. 3, two EPs merge to one hybrid EP [90,92,165] and locate at $\left(k_{x}, k_{y}\right)=(0, \pi)$ for $\gamma^{2}=(m+t)^{2}+t^{2}$ and at $\left(k_{x}, k_{y}\right)=$ $(\pi, \pi)$ for $\gamma^{2}=(m-t)^{2}+t^{2}$; the EP can be checked by substituting $\left(k_{x}, k_{y}\right)$ into $\mathcal{H}(\mathbf{k})$. The band structures in the gapless phase with different EP distributions are demonstrated in Figs. 2(b)-2(e).

The white region in the phase diagram (Fig. 3) indicate the topologically trivial phase with zero Chern number; topologically protected edge states are absent in this phase under $\mathrm{OBC}$ in either direction. Due to the large imaginary part of energy bands at large non-Hermiticity of $\gamma^{2}>(|m|+t)^{2}+t^{2}$, the energy bands are fully complex and separable. The green region also represent a phase with zero Chern number; however, in-gap topologically protected edge states exist under OBC in the $y$ direction; this point is elucidated in Sec. IV.

To characterize the topological properties of the inversion symmetric non-Hermitian Chern insulator, it is convenient to transform the Bloch Hamiltonian $\mathcal{H}(\mathbf{k})$ into a two-band model. After a similar transformation

$$
\mathcal{M}=\left(\begin{array}{cccc}
\sqrt{\mu} & -i \sqrt{\mu} & 0 & 0 \\
-i \sqrt{\nu} & \sqrt{\nu} & 0 & 0 \\
0 & 0 & \sqrt{\nu} & -i \sqrt{\nu} \\
0 & 0 & -i \sqrt{\mu} & \sqrt{\mu}
\end{array}\right)
$$

is performed, the Bloch Hamiltonian of the non-Hermitian Chern insulator changes into

$$
\mathcal{M H}(\mathbf{k}) \mathcal{M}^{-1}=\left(\begin{array}{cccc}
t \sin k_{x} & \sqrt{\mu \nu} & 0 & t e^{i k_{y}} \\
\sqrt{\mu \nu} & -t \sin k_{x} & t & 0 \\
0 & t & t \sin k_{x} & \sqrt{\mu \nu} \\
t e^{-i k_{y}} & 0 & \sqrt{\mu \nu} & -t \sin k_{x}
\end{array}\right),
$$

which possesses a two-site unit cell instead of a four-site one because of the repeated diagonal $2 \times 2$ matrix $\sqrt{\mu \nu} \sigma_{x}+$ $\left(t \sin k_{x}\right) \sigma_{z}$. This is in accordance with the spectrum presented in Fig. 2, where two upper (lower) bands can form a single band after one of them shifts by $2 \pi$ in the $k_{y}$ direction.

The equivalent two-band Bloch Hamiltonian $h(\mathbf{k})=\mathbf{B} \cdot \sigma$ obtained from $\mathcal{H}(k)$ after a similar transformation [Eq. (9)] reads

$$
h(\mathbf{k})=B_{x} \sigma_{x}+B_{y} \sigma_{y}+B_{z} \sigma_{z}
$$

The Bloch Hamiltonian is a spin-1/2 in a complex effective magnetic field $\mathbf{B}=\left(B_{x}, B_{y}, B_{z}\right)$ with $B_{x}=\sqrt{\mu \nu}+t \cos k_{y}$, $B_{y}=-t \sin k_{y}, B_{z}=t \sin k_{x}$; and $\sigma=\left(\sigma_{x}, \sigma_{y}, \sigma_{z}\right)$. In the Hermitian limit $\gamma=0$, the effective magnetic field $B_{x}=m+$ $t \cos k_{x}+t \cos k_{y}, B_{y}=-t \sin k_{y}, B_{z}=t \sin k_{x}$ is real, and $h(\mathbf{k})$ follows the Qi-Wu-Zhang model [166]. Notably, $k_{y}$ in the two-band $h(\mathbf{k})$ corresponds to $2 k_{y}$ in the four-band $\mathcal{H}(\mathbf{k})$. The energy bands of $h(\mathbf{k})$ are depicted in Appendix A as 
a comparison with the corresponding energy bands of $\mathcal{H}(\mathbf{k})$ depicted in Fig. 2.

We emphasize that $\mu \nu=0$ is the HDELs of the four-band Bloch Hamiltonian $\mathcal{H}(\mathbf{k})$. In contrast, the equivalent two-band Bloch Hamiltonian $h(\mathbf{k})$ is Hermitian at $\mu \nu=0$; thus, the HDELs are absent in $h(\mathbf{k}) . \mathcal{H}(\mathbf{k})$ and $h(\mathbf{k})$ are related by the similar transformation $\mathcal{M}$ at $\mu \nu \neq 0$; they are not related by the similar transformation $\mathcal{M}$ at $\mu \nu=0\left(\mathcal{M}^{-1}\right.$ does not exits $)$ although their energy bands are identical. In this sense, the HDELs are removed and absent in $h(\mathbf{k})$. Notably, the wavefunction singularity in $\mathcal{H}(\mathbf{k})$ does not appear at the HDELs; thus, the equivalent two-band Bloch Hamiltonian $h(\mathbf{k})$ carries the topological properties of $\mathcal{H}(\mathbf{k})$.

Chern number in the gapped phase. The Bloch Hamiltonian $h(\mathbf{k})$ describes an RM ladder with glider reflection symmetry $[167,168]$ consisting of two coupled RM chains, and the interladder leg coupling is $\sqrt{\mu \nu}$. In the regions that the energy bands are separated [92], the Chern number for the energy band is a topological invariant that characterizes the topological properties and the appearance of edge states of the system under $\mathrm{OBC}$.

The eigenvalue is $\varepsilon_{ \pm}(\mathbf{k})= \pm B$, where $B=\left(B_{x}^{2}+B_{y}^{2}+\right.$ $\left.B_{z}^{2}\right)^{1 / 2}$; the corresponding eigenstate is $\left|\psi_{ \pm}(\mathbf{k})\right\rangle=N_{ \pm}^{-1}\left[B_{z} \pm\right.$ $\left.B, B_{x}+i B_{y}\right]^{T}$, where $N_{ \pm}=\left(\left|B_{z} \pm B\right|^{2}+\left|B_{x}+i B_{y}\right|^{2}\right)^{1 / 2}$ and the eigenstate $\left|\psi_{ \pm}(\mathbf{k})\right\rangle$ satisfies $\left\langle\psi_{ \pm}(\mathbf{k}) \mid \psi_{ \pm}(\mathbf{k})\right\rangle=1$. The wave-function singularity occurs at $B_{z} \pm B=B_{x}+i B_{y}=0$ [4] (Notably, the wavefunction singularity is not refer to as the EP, where eigenstate coalescence). The Berry connection for the eigenstate is $A_{ \pm}(\mathbf{k})=-i\left\langle\phi_{ \pm}(\mathbf{k})\left|\nabla_{\mathbf{k}}\right| \psi_{ \pm}(\mathbf{k})\right\rangle$ $[60,61,63]$, and the Berry curvature is $\Omega_{ \pm}(\mathbf{k})=\nabla_{\mathbf{k}} \times A_{ \pm}(\mathbf{k})$, where $\left|\phi_{ \pm}(\mathbf{k})\right\rangle$ is the eigenstate of $h^{\dagger}(\mathbf{k})$ with corresponding energy bands $\varepsilon_{ \pm}^{*}(\mathbf{k})$. The eigenstates of $h(\mathbf{k})$ and $h^{\dagger}(\mathbf{k})$ constitute a biorthogonal basis. $\left|\psi_{ \pm}(\mathbf{k})\right\rangle$ and $\left|\phi_{ \pm}(\mathbf{k})\right\rangle$ are known as the right and left eigenstates, and they satisfy biorthogonal condition $\left\langle\phi_{ \pm}(\mathbf{k}) \mid \psi_{ \pm}\left(\mathbf{k}^{\prime}\right)\right\rangle=\delta_{\mathbf{k k}^{\prime}}[169,170]$. The biorthogonal condition does not settle the normalization coefficients of the left and right eigenstates; associated with the normalization of right eigenstate $\left\langle\psi_{ \pm}(\mathbf{k}) \mid \psi_{ \pm}(\mathbf{k})\right\rangle=1$, the normalization coefficients of eigenstates are fixed. The Chern number is defined as the integration of $\Omega_{ \pm}(\mathbf{k})$ over the whole first BZ

$$
C_{ \pm}=\frac{1}{2 \pi} \iint_{\mathrm{BZ}} d k_{x} d k_{y} \Omega_{ \pm}
$$

The Chern number can be alternatively defined from other choice of Berry curvature based on the right and left eigenstates $\Omega_{ \pm}^{R L}(\mathbf{k})=-i \nabla_{\mathbf{k}} \times\left[\left\langle\psi_{ \pm}(\mathbf{k})\left|\nabla_{\mathbf{k}}\right| \phi_{ \pm}(\mathbf{k})\right\rangle\right]$, solely based on the right eigenstate $\Omega_{ \pm}^{R R}(\mathbf{k})=-i \nabla_{\mathbf{k}} \times$ $\left[\left\langle\psi_{ \pm}(\mathbf{k})\left|\nabla_{\mathbf{k}}\right| \psi_{ \pm}(\mathbf{k})\right\rangle\right]$, and solely based on the left eigenstate $\Omega_{ \pm}^{L L}(\mathbf{k})=-i \nabla_{\mathbf{k}} \times\left[\left\langle\phi_{ \pm}(\mathbf{k})\left|\nabla_{\mathbf{k}}\right| \phi_{ \pm}(\mathbf{k})\right\rangle\right]$. Notably, the four definitions of Chern numbers are equivalent $[92,147]$.

The Chern numbers for the upper and lower bands are opposite $C \equiv C_{-}=-C_{+}$. The Chern number for either band is capable of characterizing the topological properties of the corresponding phases. The Chern number different between two bands directly reflects the number of edge modes at the interface of two distinct bulks. At large non-Hermiticity, $\mu \nu<$ 0 , the Berry curvature $\Omega_{ \pm}(\mathbf{k})$ is well-defined in the BZ. The wave-function singularity results in a nonzero Chern number, which predicts the nontrivial topology and the existence
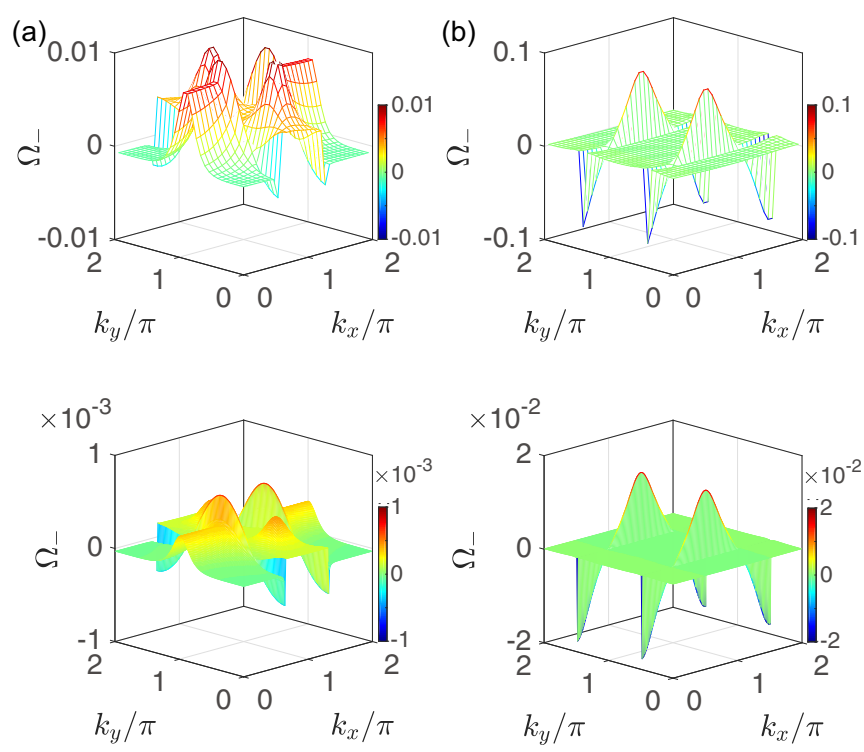

FIG. 4. Berry curvature in the BZ. Chern number as a summation of the Berry curvature in the entire BZ yields $C=1.00$ for (a) $\gamma=$ $1 / 2$ and $C=0.00$ for (b) $\gamma=5 / 2$ at $m=t=1$. The BZ is equally cut into $30 \times 30(150 \times 150)$ pieces in the numerical calculation for the upper (lower) panel. At $k_{x}=2 \pi / 3,4 \pi / 3$ in (a), $\mu \nu=0$ and $h(\mathbf{k})$ is Hermitian; $\mu \nu=0$ is absent in (b).

of edge states. For the wave function with singularity, two gauges are used to describe the wave function. $\left|\psi_{ \pm}^{\text {II }}(\mathbf{k})\right\rangle$ replaces $\left|\psi_{ \pm}^{\mathbf{I}}(\mathbf{k})\right\rangle$ in an area $D$ that encloses the singularity of $\left|\psi_{ \pm}^{\mathbf{I}}(\mathbf{k})\right\rangle$, that is, $\left|\psi_{ \pm}^{\mathbf{I I}}(\mathbf{k})\right\rangle=\left|\psi_{ \pm}^{\mathbf{I}}(\mathbf{k})\right\rangle e^{i \varphi_{ \pm}^{R}(\mathbf{k})},\left|\phi_{ \pm}^{\mathbf{I I}}(\mathbf{k})\right\rangle=$ $\left|\phi_{ \pm}^{\mathbf{I}}(\mathbf{k})\right\rangle e^{i \varphi_{ \pm}^{L}(\mathbf{k})}$. The phase dependence between two gauges results in a relation between two Berry connections $A_{ \pm}^{\text {II }}(\mathbf{k})=$ $A_{ \pm}^{\mathbf{I}}(\mathbf{k})+\nabla_{\mathbf{k}} \varphi_{ \pm}^{R}(\mathbf{k})$. The Stokes' theorem indicates that the Chern number equals the winding of the variation of $\varphi_{ \pm}^{R}$ along the loop that encloses the area $D$.

The Berry curvature $\Omega_{ \pm}(\mathbf{k})$ is ill-defined at $\mu \nu=0$, where eigenstates of $\mathcal{H}(\mathbf{k})$ coalesce in pairs and one-half of eigenstates vanish. Notably, $\sqrt{\mu \nu}$ changes between real and imaginary as $k_{x}$ crosses $\mu \nu=0$ in the BZ. Consequently, $B_{x}\left(h_{x, \pm}\right)$ is not smooth and the Berry curvature $\Omega_{ \pm}(\mathbf{k})$ is ill-defined at $\mu \nu=0$. However, the Chern number is an integral of the Berry curvature in the entire BZ, the ill-defined Berry curvatures are only a finite number of lines; and the wave-function singularity does not appear at $\mu \nu=0$. Thus the Chern number determined by the wave-function singularity in the BZ is not affected by the presence of ill-defined Berry curvatures at $\mu \nu=0$. The nonzero (zero) Chern number $C= \pm 1$ (0) is verified from the numerical simulation in the discretized BZ [171]. The numerical results of the Berry curvature are exemplified in Fig. 4 for the topologically nontrivial phase with $C=1$ and the topologically trivial phase with $C=0$. In Fig. $4(\mathrm{a}), \mu \nu=0$ is the boundary between Hermitian and non-Hermitian $h(\mathbf{k}): h(\mathbf{k})$ is Hermitian when $\mu \nu>0$, but is non-Hermitian when $\mu \nu<0$.

Winding number in the gapless phase. In the gapless phase, the Chern number is not available, we define a vector field $\mathbf{F}_{ \pm}=\left(\left\langle\sigma_{x}\right\rangle_{ \pm},\left\langle\sigma_{y}\right\rangle_{ \pm},\left\langle\sigma_{z}\right\rangle_{ \pm}\right)$to characterize the topological features of the gapless phase. The vector field $\mathbf{F}_{ \pm}$is defined 


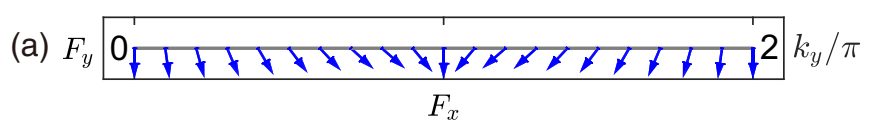

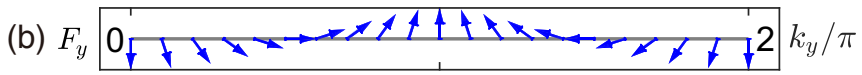
$F_{x}$

(c) $F_{z} \frac{\theta+1+1+2}{F_{x}} k_{x} / \pi$

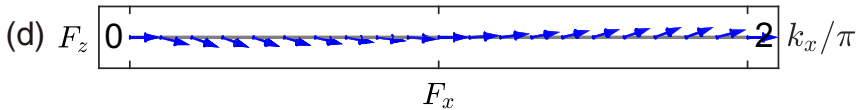

FIG. 5. The planar vector field $F_{-}$for the lower band of $h(\mathbf{k})$. (a) $\gamma=3 / 2, k_{x}=0$; (b) $\gamma=2, k_{x}=0$; (c) $\gamma=3 / 2, k_{y}=\pi$; and (d) $\gamma=2, k_{y}=\pi . F_{-}$at $k_{y}=\pi$ in $h(\mathbf{k})$ predicts the topological properties of $\mathcal{H}(\mathbf{k})$ at $k_{y}=0$. Other system parameters are $m=$ $t=1$. (a) and (d) correspond to $w_{-}=0$; (b) and (c) correspond to $w_{-}=1$.

by the average values of Pauli matrices: $F_{ \pm, x, y, z}=\left\langle\sigma_{x, y, z}\right\rangle_{ \pm}=$ $\left\langle\psi_{ \pm}(\mathbf{k})\left|\sigma_{x, y, z}\right| \psi_{ \pm}(\mathbf{k})\right\rangle$ and the subscript \pm indicate the index of the energy band. Under the normalization of the right eigenstate $\left\langle\psi_{ \pm}(\mathbf{k}) \mid \psi_{ \pm}(\mathbf{k})\right\rangle=1$, the amplitude of the vector field is unity, that is, $\left|\mathbf{F}_{ \pm}\right|^{2}=1$. The vector field is depicted in Fig. 5 to elucidate that a winding number associated with $\mathbf{F}_{ \pm}$accurately predicts the (non)existence of edge states in the gapless phase. For the system under OBC in the $y$ direction [Fig. 6(b)], the trivial and nontrivial windings of the planar vector field $\mathbf{F}_{ \pm, x y}=\left(\left\langle\sigma_{x}\right\rangle_{ \pm},\left\langle\sigma_{y}\right\rangle_{ \pm}\right)$as $k_{y}$ varying a period predict the attaching point of the edge states and the bulk states in the complex energy bands

$$
w_{ \pm}=(2 \pi)^{-1} \int_{0}^{2 \pi} d k_{y} \nabla_{k_{y}} \phi_{ \pm}
$$

where $\tan \phi_{ \pm}=F_{ \pm, y} / F_{ \pm, x}$. The winding numbers of two energy bands are identical, that is, $w_{+}=w_{-}$. The nontrivial $2 \pi$ varying direction accumulation of the planar vector field $\mathbf{F}_{ \pm, x z}=\left(\left\langle\sigma_{x}\right\rangle_{ \pm},\left\langle\sigma_{z}\right\rangle_{ \pm}\right)$in a period of $k_{x}$ predicts the edge states for the system under OBC in the $x$ direction [Fig. 6(a)]; correspondingly, $w_{ \pm}=(2 \pi)^{-1} \int_{0}^{2 \pi} d k_{x} \nabla_{k_{x}} \phi_{ \pm}$with $\tan \phi_{ \pm}=$
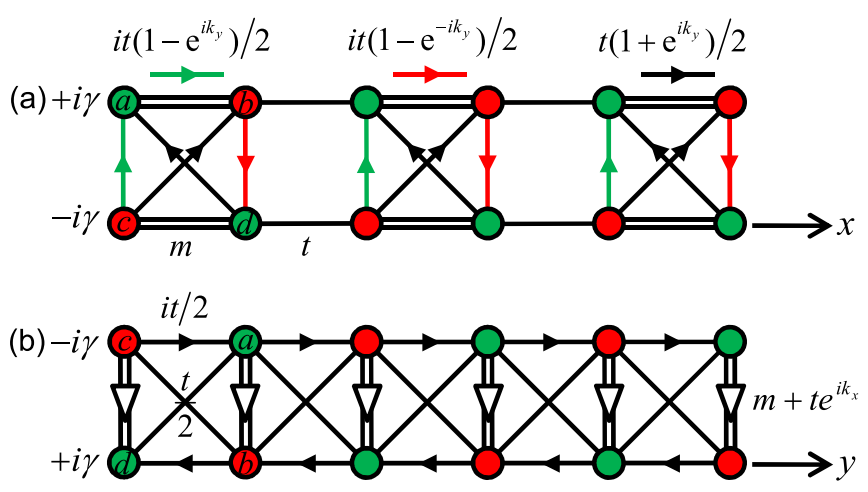

FIG. 6. (a) [(b)] Schematic of the 1D projection at set $k_{y}\left(k_{x}\right)$ of the 2D inversion symmetric non-Hermitian Chern insulator. (a) Quasi-1D SSH ladder. (b) Quasi-1D Creutz ladder. The green (red) solid circle indicates the site with gain (loss).
$F_{ \pm, z} / F_{ \pm, x}$ and $w_{ \pm}$predicts the topological phase transition at $k_{y}=\pi / 2$. Notably, the topological phase transition at $k_{y}=$ $\pi / 2$ in $h(\mathbf{k})$ indicates the existence of edge states in the region $k_{y}=[-\pi, \pi]$ as depicted in Fig. 7 for $\mathcal{H}$ under OBC in the $x$ direction. This is because the folded $\mathrm{BZ}$ in the $k_{y}$ direction of $h(\mathbf{k})$ yields the $\mathrm{BZ}$ of $\mathcal{H}(\mathbf{k})$.

\section{ENERGY BANDS AND EDGE STATES OF THE EDGE HAMILTONIAN}

The bulk topology of the system determines the phase diagram, which accurately predicts the topological phase transition and the (non)existence of edge states in different topological phases. In this section, we elucidate the role played by the Chern number for the gapped phase and the winding number for the gapless phase in the topological characterization through the investigation of the energy bands and edge states under different $\mathrm{OBC}$ only in the $x$ and $y$ directions, respectively. The $\mathrm{PBC}$ and $\mathrm{OBC}$ spectra are not dramatically different from each other owing to the validity of conventional bulk-boundary correspondence protected by the inversion symmetry.

$1 D$ edge Hamiltonian under $O B C$. The system under $\mathrm{OBC}$ is referred to the edge Hamiltonian because it is generated by truncating the bulk Hamiltonian in a certain way. The two-dimensional Chern insulator reduces to a non-Hermitian quasi-1D SSH ladder or quasi-1D Creutz ladder at a fixed momentum in the $y$ or $x$ direction (Fig. 6), respectively. The non-Hermitian quasi-1D Creutz ladder is equivalent to a 1D RM chain with asymmetric couplings presented alternately (see Sec. V for more detail).

For the Chern insulator under $\mathrm{PBC}$ in the $y$ direction and OBC in the $x$ direction, we apply the Fourier transformation in the $y$ direction. This projection gives a quasi-1D SSH ladder with momentum $k_{y}$-dependent couplings and staggered on-site potentials, as presented in Fig. 6(a). Similarly, for the Chern insulator under PBC in the $x$ direction and OBC in the $y$ direction, the projection lattice reduces to a quasi-1D Creutz ladder with a momentum $k_{x}$-dependent couplings in the inter ladder, as presented in Fig. 6(b).

We study the inversion symmetric non-Hermitian Chern insulator under $\mathrm{PBC}$ and $\mathrm{OBC}$ in the $y$ and $x$ directions, respectively. The upper panel of Fig. 7 depicts the spectra under $\mathrm{PBC}$ in the $x$ direction and under OBC in the $y$ direction; the lower panel of Fig. 7 depicts the spectra under PBC in the $y$ direction and under $\mathrm{OBC}$ in the $x$ direction at the same system parameters as those in the upper panel of Fig. 7.

Gapped phase. In the gapped phase, the Chern number is the topological invariant. The nonzero Chern numbers of the upper and lower bands in the topologically nontrivial phase indicate the existence of a pair of topologically protected helical edge states under OBC. The two helical edge states in a pair localize at the left and right edges of the 1D system, respectively. The white region with zero Chern number are topologically trivial phases without topologically protected edge states. The green region with zero Chern number consists of in-gap edge states. We first discuss the topological edge states and then discuss the topological phase transition in different gapped phases. 

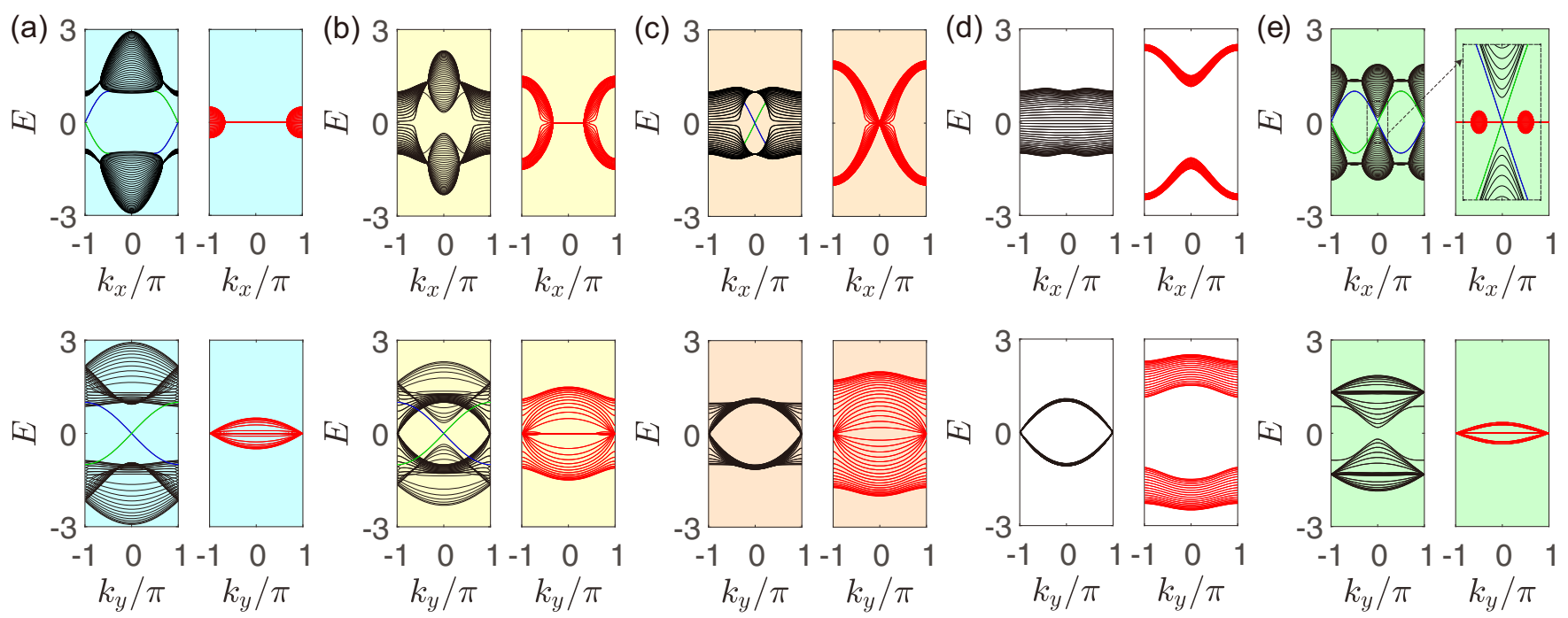

FIG. 7. Energy spectrum for $\mathcal{H}$ under OBC. The upper (lower) panel represents the energy spectra of the system under PBC in the $x(y)$ direction, but under OBC in the $y(x)$ direction; the schematic is in Fig. 6 . The parameters are (a) $\gamma=1 / 2,(\mathrm{~b}) \gamma=3 / 2$, (c) $\gamma=2$, (d) $\gamma=5 / 2$; other parameters are $m=t=1$ in (a)-(d). In (e), $m=0, t=1$, and $\gamma=1 / 2$. The band energy repetition period is $\pi$ for the upper panel of (e), where the inset on the right side are zoom-in plot of the area in the dashed box. The edge states reside inside the band gap without touching the bulk bands. The parameters in the plots are indicated by the solid dots in the phase diagram shown in Fig. 3. The black (red) line is the real (imaginary) part of band energy. The number of unit cells of 1D projection lattices is twenty.

Figure 7(a) depicts the spectrum in the topologically nontrivial phase with $C=1$; a pair of topologically protected helical edge states exist, localizing on the left and right boundaries of the 1D lattice, respectively. In Fig. 7(d), the system is in the topologically trivial region, the white region with $C=0$, the edge state is absent. The OBC spectra in the green region with $C=0$ are depicted in Fig. 7(e). In-gap edge states are observed under OBC in the $y$ direction; both edge states are detached from the upper and lower bands; this is indicated by the zero Chern number and presented in the inset of Fig. 7(e). Two in-gap edge states cross at $k_{x}=$ $0, \pi$. As non-Hermiticity increases, the in-gap edge states may touch the upper and lower bands at $\gamma \geqslant 1$ with the diminishing gap at $k_{x}= \pm \pi / 2$. By contrast, the topologically protected helical edge states are absent for the system under $\mathrm{OBC}$ in the $x$ direction in the lower panel. We generalize the polarization into the non-Hermitian region to characterize the topological in-gap edge states $[172,173]$. The polarization is defined by the Berry connection based on the left and right eigenstates; the projection in the $x(y)$ direction is $P_{ \pm, x(y)}=(2 \pi)^{-2} \iint_{\mathrm{BZ}} d k_{x} d k_{y} A_{ \pm, x(y)}(\mathbf{k})$, where $A_{ \pm, x(y)}(\mathbf{k})=$ $-i\left\langle\phi_{ \pm}(\mathbf{k})\left|\nabla_{k_{x(y)}}\right| \psi_{ \pm}(\mathbf{k})\right\rangle$. In the green region with $C=0$, the wave function has no singularities; thus, the polarization is well defined and we have the 2D Zak phases associated fractional polarization $P_{-, y}=-1 / 2$ and $P_{-, x}=0$ [172]. The in-gap edge states are topological protected, being robust to the inversion symmetric perturbations that without breaking the pseudo-Hermiticity. Two in-gap edge states remain in the band gap and are detached from the bulk bands. The details about the robustness of in-gap edge states are provided in Appendix B.

The appearance of band touching affects the (non)existence of topologically protected helical edge states. We elaborate this point by considering gapped phases in the regions of $m>0$ and $\gamma<1$. Notably, the inversion symmetric 2D
non-Hermitian Chern insulator is anisotropic. In the green region of the phase diagram, the helical edge states appear only in the system under $\mathrm{OBC}$ in the $y$ direction [upper panel of Fig. 7(e)]. Between the green and cyan regions, the system experiences a band touching with DP at $\left(k_{x}, k_{y}\right)=(0,0)$ [Fig. 2(d)]. Thus, for the system under OBC in the $y$ direction, the in-gap helical edge states crossing at $k_{x}=0, \pi$ change into helical edge states that connect the upper and lower bands and cross only at $k_{x}=\pi$ [upper panel of Fig. 7(a)]. As $m$ continuously increases, the system experiences a band touching with DP at $\left(k_{x}, k_{y}\right)=(\pi, 0)$ and the helical edge states in the cyan region crossing at $k_{x}=\pi$ are destroyed and disappear in the white region. Similarly, for the system under $\mathrm{OBC}$ in the $x$ direction, the helical edge states crossing at $k_{y}=$ 0 are formed after the system comes across the band touching with DP at $\left(k_{x}, k_{y}\right)=(0,0)$ and enters the cyan region in the phase diagram. As $m$ continuously increases, the system experiences a band touching with DP at $\left(k_{x}, k_{y}\right)=(\pi, 0)$ and the helical edge states in the cyan region crossing at $k_{y}=0$ are destroyed and disappear in the white region.

Gapless phase. In the gapless phase, the helical edge states appear only under $\mathrm{OBC}$ in one direction and are predicted by the winding number $w_{ \pm}$associated with the vector field $\mathbf{F}_{ \pm}$. The nonzero winding of the vector field predicts the presence of edge states detaching the bulk band. The planar vector field $\mathbf{F}_{-, x y}$ is depicted in Figs. 5(a) and 5(b). The winding number is $w_{-}=0$ for Fig. 5(a) and is $w_{-}=1$ for Fig. 5(b), which predict the absence and presence of edge states at $k_{x}=0$ for the system under $\mathrm{OBC}$ in the $y$ direction in the upper panel of Figs. 7(b) and 7(c), respectively. The planar vector field $\mathbf{F}_{-, x z}$ in Figs. 5(c) and 5(d) are depicted. The winding number is $w_{-}=1$ for Fig. 5(c) and is $w_{-}=0$ for Fig. 5(d), which predict the presence and absence of edge states at $k_{y}=0$ for the system under OBC in the $x$ direction in the lower panel of Figs. 7(b) and 7(c), respectively. 
Topologically protected helical edge states exist in the orange region of the phase diagram in the gapless phase only for the $\mathrm{OBC}$ in the $y$ direction. We consider a process with increasing non-Hermiticity. The system first experiences a nontrivial phase with $C=1$ in the cyan region, then it enters the gapless phase, and finally it stays in the trivial phase with $C=0$ in the white region. For the system under OBC in the $y$ direction, the helical edge states cross at $k_{x}=\pi$. As non-Hermiticity increases, the system enters the gapless phase in the yellow region from the cyan region. The EPs appear at $\left(k_{x}, k_{y}\right)=(\pi, \pi)$ and then the $k_{x}$ position of EPs changes as $\gamma$ increases. Thus the helical edge states crossing at $k_{x}=\pi$ are destroyed by non-Hermiticity [upper panel of Fig. 7(b)]; the crossing at $k_{x}=\pi$ disappear. However, the DP appears at $\left(k_{x}, k_{y}\right)=(0,0)$ at increasing $\gamma$; then the helical edge states cross at $k_{x}=0$ and reappear until the EPs move to $\left(k_{x}, k_{y}\right)=(0, \pi)$, which destroys the helical edge states once again. Thereafter, the system enters the trivial phase with $C=0$ in the white region. For instance, as non-Hermiticity increases to $\gamma=\sqrt{3}$ for $m=t=1$, an additional band touching DP is formed at $k_{x}=0$. Therefore two helical edge states reappear in the orange region of the phase diagram at large non-Hermiticity as shown in the upper panel of Fig. 7(c); they cross at $k_{x}=0$ instead of at $k_{x}=\pi$ as in the $C=1$ [upper panel of Fig. 7(a)]. At even larger $\gamma=5 / 2$ in Fig. 7(d), the system is in the topologically trivial region without the presence of edge states.

By contrast, the topologically protected helical edge states exist in the yellow region of the phase diagram in the gapless phase only for the OBC in the $x$ direction as elucidated in the lower panel of Fig. 7. For the system under OBC in the $x$ direction, the helical edge states cross at $k_{y}=0$ [lower panel of Fig. 7(a)]. The band touching EPs are fixed at $k_{y}=\pi$ independent of non-Hermiticity, and do not affect the helical edge states until the DP appears at $\left(k_{x}, k_{y}\right)=(0,0)$ [Fig. 2(d)]. Then, the helical edges crossing at $k_{y}=0$ are destroyed and disappear in the gapless phase in the orange region [lower panel of Fig. 7(c)]. The gapless phase with band touching DP is the boundary for the appearance of gapless topologically protected edge states.

Edge states. For the system under $\mathrm{OBC}$ in the $y$ direction, the left edge state localizes at the left boundary and has eigenenergy $E_{L}\left(k_{x}\right)=-t \sin k_{x}$. We consider $\psi_{1}=1$ for convenience without loss of generality. The wave functions of the left edge state $\left|\psi_{L}\right\rangle$ satisfies the recursion relation

$$
\left\{\begin{aligned}
\psi_{2 j} & =-i \psi_{2 j-1}, \\
\psi_{2 j+1} & =\left[(-1)^{j} \gamma / t-\left(m+t \cos k_{x}\right) / t\right] \psi_{2 j-1},
\end{aligned}\right.
$$

where $j=1,2,3, \ldots, n / 2-1$ is the index, and $\psi_{n}=$ $-i \psi_{n-1}$. The right edge state $\left|\psi_{R}\right\rangle$, localizes at the right boundary and has eigenenergy $E_{R}\left(k_{x}\right)=t \sin k_{x}$. We can consider $\psi_{n}=1$, and the recursion relation for the wave functions of the right edge state $\left|\psi_{R}\right\rangle$ is

$$
\left\{\begin{aligned}
\psi_{n-(2 j-1)} & =-i \psi_{n-(2 j-2)}, \\
\psi_{n-2 j} & =\left[(-1)^{j} \gamma / t-\left(m+t \cos k_{x}\right) / t\right] \psi_{n-(2 j-2)},
\end{aligned}\right.
$$

where $j=1,2,3, \ldots, n / 2-1$ is the index, and $\psi_{1}=-i \psi_{2}$. Notably, the edge state energy is independent of the coupling

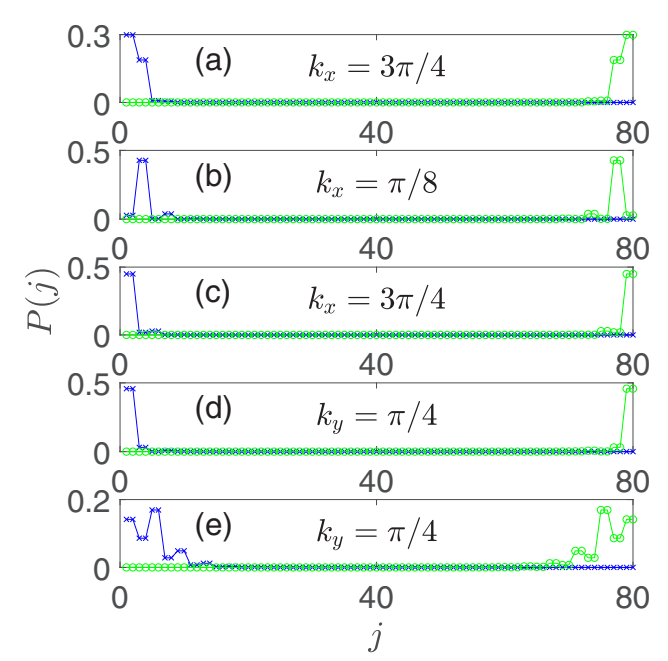

FIG. 8. Edge states for the quasi-1D lattice in Fig. 6 under OBC. The set momentum in the direction under PBC is marked inside. The parameters are identical with those chosen in Fig. 7. $\gamma=1 / 2$ in (a), (c), and (d); $\gamma=2$ in (b); and $\gamma=3 / 2$ in (e). Other parameters are $m=t=1$ except for $m=0, t=1$ in (c).

$m$ and the non-Hermiticity $\gamma$ although both affect the wave functions. The edge states under OBC in the $y$ direction in different topological phases are depicted in Figs. 8(a)-8(c). Figure 8 (a) presents the edge states in the $C=1$ phase, as illustrated in Fig. 7(a); Fig. 8(b) represents the edge states in the gapless phase, as illustrated in Fig. 7(c); Fig. 8(c) represents the in-gap edge states in the $C=0$ phase, as illustrated in Fig. 7(e). The green region $(C=0)$ with in-gap edge states is a novel phase induced by non-Hermiticity.

For the system under OBC in the $x$ direction, the left edge state localizes at the left boundary and has eigenenergy $E_{L}\left(k_{y}\right)=-t \sin \left(k_{y} / 2\right)$. The wave functions of the left edge state $\left|\psi_{L}\right\rangle$ satisfies the recursion relation

$$
\left\{\begin{array}{l}
\psi_{4 j+1}=\frac{i \gamma}{t} \psi_{4 j-1}-\left[\frac{m}{t}-\cos \left(\frac{k_{y}}{2}\right)\right] \psi_{4 j-3}, \\
\psi_{4 j+3}=-\frac{(i \gamma / t) \psi_{4 j+1}}{m / t+\cos \left(k_{y} / 2\right)}-\frac{\psi_{4 j-1}}{m / t+\cos \left(k_{y} / 2\right)} \\
\psi_{4 j+2}=\psi_{4 j+1} e^{i \theta} \\
\psi_{4 j+4}=-\psi_{4 j+3} e^{i \theta}
\end{array}\right.
$$

where $j=1,2,3, \ldots, n / 4-1$ is the index, and $\psi_{1}=$ $1, \quad \psi_{2}=e^{i \theta}, \quad \psi_{3}=-(i \gamma / t) /\left[m / t+\cos \left(k_{y} / 2\right)\right]$, and $\psi_{4}=$ $-\psi_{3} e^{i \theta}$ with $\sin \theta=\sin \left(k_{y} / 2\right)$, and $\cos \theta=-\cos \left(k_{y} / 2\right)$. The right edge state, localizes at the right boundary and has eigenenergy $E_{R}\left(k_{y}\right)=t \sin \left(k_{y} / 2\right)$. The recursion relation for the wave functions of the right edge state $\left|\psi_{R}\right\rangle$ is

$$
\left\{\begin{aligned}
\psi_{n-4 j} & =\frac{i \gamma}{t} \psi_{n-(4 j-2)}-\left[\frac{m}{t}-\cos \left(\frac{k_{y}}{2}\right)\right] \psi_{n-(4 j-4)} \\
\psi_{n-(4 j+2)} & =-\frac{(i \gamma / t) \psi_{n-4 j}}{m / t+\cos \left(k_{y} / 2\right)}-\frac{\psi_{n-(4 j-2)}}{m / t+\cos \left(k_{y} / 2\right)} \\
\psi_{n-(4 j+1)} & =\psi_{n-4 j} e^{i \theta} \\
\psi_{n-(4 j+3)} & =-\psi_{n-(4 j+2)} e^{i \theta}
\end{aligned}\right.
$$

where $j=1,2,3, \ldots, n / 4-1$ is the index, and $\psi_{n}=1$, $\psi_{n-1}=e^{i \theta}, \psi_{n-2}=-(i \gamma / t) /\left[m / t+\cos \left(k_{y} / 2\right)\right]$, and $\psi_{n-3}=$ $-\psi_{n-2} e^{i \theta}$ with $\sin \theta=\sin \left(k_{y} / 2\right)$, and $\cos \theta=-\cos \left(k_{y} / 2\right)$. The edge states under OBC in the $x$ direction in different 


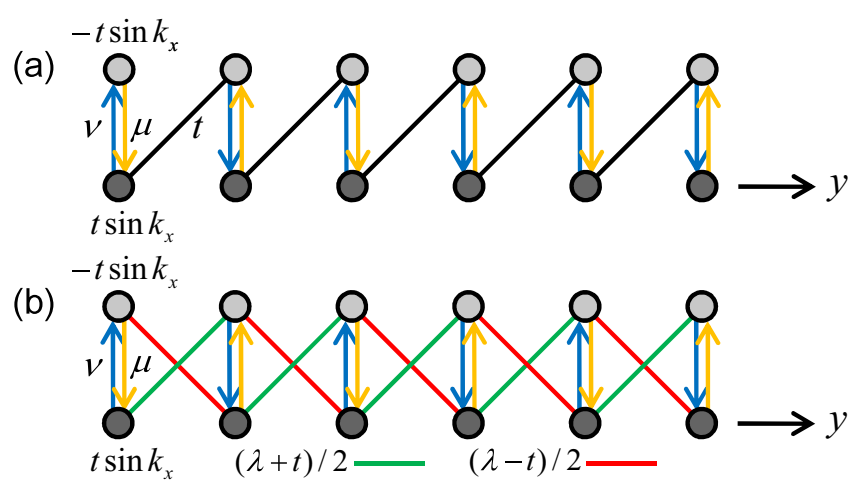

FIG. 9. (a) 1D RM chain. (b) Quasi-1D RM ladder with asymmetric intra-ladder leg coupling at $\lambda \neq \pm t$. (b) can be regarded as a 1D RM chain of (a) with symmetric long-range coupling. $-t \sin k_{x}$ and $t \sin k_{x}$ are the on-site real potentials for the upper (light gray) and lower (dark gray) sites, respectively. The non-Hermitian element is the asymmetric coupling $\mu-v$.

topological phases are depicted in Figs. 8(d) and 8(e). Figure $8(\mathrm{~d})$ represents the edge states in the $C=1$ phase, as illustrated in Fig. 7(a); Fig. 8(e) represents the edge states in the gapless phase, as illustrated in Fig. 7(b).

\section{DISCUSSION}

$1 D$ projection of the Chern insulator. The quasi-1D Creutz ladder [Fig. 6(b)] changes into a 1D RM ladder with asymmetric couplings [Fig. 9(a)] after applying a uniform transformation to each dimer (the upper and lower sites with intradimer coupling $m), I_{2 n} \otimes\left(\sigma_{0}+i \sigma_{x}\right) / \sqrt{2}$. Moreover, if the off-diagonal coupling strength in the plaquette is $\lambda / 2$ instead of $t / 2$ in Fig. 1, we obtain a quasi-1D RM ladder presented in Fig. 9(b), which can be regarded as a 1D RM chain with longrange coupling or as two coupled RM chains with asymmetric interchain coupling. The coupling depicted in green (red) is the nearest-neighbor coupling between asymmetric dimers; the red (green) coupling is taken as the long-range coupling, studied in Ref. [133]. In the situation that $\lambda=t(\lambda=-t)$, the long-range couplings vanish. In the neighboring asymmetric dimers, the asymmetric couplings with stronger and weaker coupling amplitudes are in the opposite directions, which is due to the difference in the one-way amplification and oneway attenuation. At $\mu \nu=0$, the asymmetric couplings are unidirectional $[163,174]$ and result in the HDELs. Notably, alternately introducing the gain and loss under inversion symmetry prevents the one-way amplification, one-way attenuation, and nonzero accumulation of imaginary flux. This is the key point for the validity of conventional bulk-boundary correspondence.

Experimental realization. The non-Hermitian Chern insulator can be simulated by dissipative ultracold atoms in an optical lattice with a synthetic magnetic field and the spinorbital coupling [175-177]. In addition, the non-Hermitian Chern insulator can be implemented in optical and photonic systems such as coupled waveguide and coupled resonator lattices [178,179], where optical dissipation and radiation ubiquitously exist. Instead of incorporating a balanced gain and loss, introducing different dissipations in different sublat- tices facilitates realization of passive non-Hermitian topological systems in experiment. It is convenient to induce losses by sticking additional absorption materials. In Ref. [129], proposed realization of a coupled resonator optical waveguide lattice of the non-Hermitian Creutz ladder with gain and loss; this proposal is directly applicable realizing the inversion symmetric non-Hermitian Chern insulator investigated in this study by coupling the Creutz ladders together and adding the non-Hermiticity alternately in the $x$ and $y$ directions.

\section{SUMMARY}

In summary, we investigated an inversion symmetric 2D non-Hermitian Chern insulator with balanced gain and loss in $x$ and $y$ directions and found that the conventional bulkboundary correspondence holds. The bulk topology determines the phase diagram and accurately predicts the topological phase transition and the (non)existence of topological edge states for the system under OBC. The helical edge states exist in the phase with nonzero Chern number for the system under $\mathrm{OBC}$ in both directions. By contrast, non-Hermiticity can vary the system topology and destroy (create) helical edge states. Non-Hermiticity creates topological gapless phase, where the helical edge states exist in the inversion symmetric nonHermitian Chern insulator under OBC in only one direction. The winding number associated with the vector field of the average values of Pauli matrices predicts the edge states in the gapless phase. Furthermore, non-Hermiticity creates a novel topological phase with zero Chern number, in which a pair of topologically protected in-gap helical edge states are found, protected by the 2D Zak phase associated fractional polarization; this feature differs from the trivial phase with zero Chern number and without edge states. Our findings provide insights into symmetry-protected non-Hermitian topological insulators.

\section{ACKNOWLEDGMENTS}

This work was supported by National Natural Science Foundation of China (Grants No. 11975128, No. 11605094, and No. 11874225) and the Fundamental Research Funds for the Central Universities, Nankai University (Grants No. 63191522 and No. 63191738).

\section{APPENDIX A: ENERGY BAND OF THE TWO-BAND EQUIVALENT HAMILTONIAN}

We show the energy band of the equivalent two-band Bloch Hamiltonian $h(\mathbf{k})$ in Fig. 10 as the comparison with the energy bands of the four-band Bloch Hamiltonian $\mathcal{H}(\mathbf{k})$ depicted in Fig. 2. Three typical phases are presented. In Fig. 10(a), we show the topologically nontrivial phase with $C=1$ to compare with the energy bands depicted in Fig. 2(a). In Fig. 10(b), we show the gapless phase to compare with the energy bands depicted in Fig. 2(d). In Fig. 10(c), we show the topologically trivial phase with $C=0$ to compare with the energy bands depicted in Fig. 2(f). 

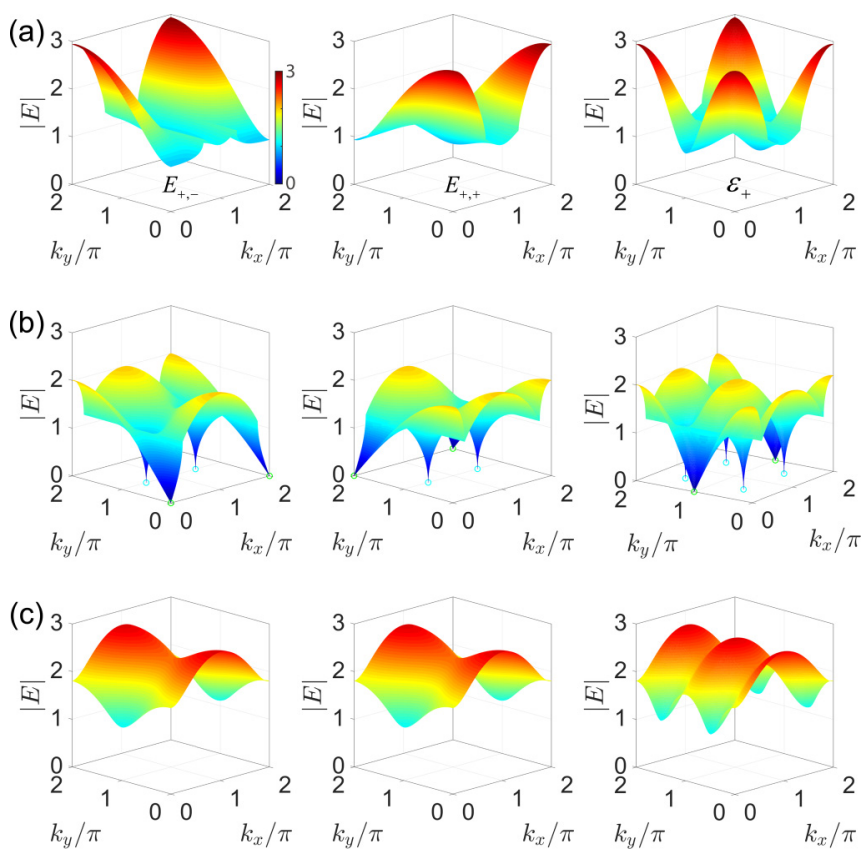

FIG. 10. Two upper bands $E_{+, \pm}$of $\mathcal{H}(\mathbf{k})$ are depicted in the left and middle panels; the upper band $\varepsilon_{+}$of $h(\mathbf{k})$ is depicted in the right panel. The parameters are identical to Figs. 2(a), 2(d) and 2(f). $m=$ $t$; (a) $\gamma=1 / 2$, (b) $\gamma=\sqrt{3}$, and (c) $\gamma=5 / 2$. The EPs (DPs) are marked by the cyan (green) circles.

\section{APPENDIX B: ROBUSTNESS OF IN-GAP EDGE STATE}

We consider the 2D Chern insulator under OBC in the $y$ direction as schematically illustrated in Fig. 6(b). The ingap edge states are robust to the inversion symmetric perturbations. We demonstrate the robustness to the inversion symmetric perturbations in the form of on-site potentials
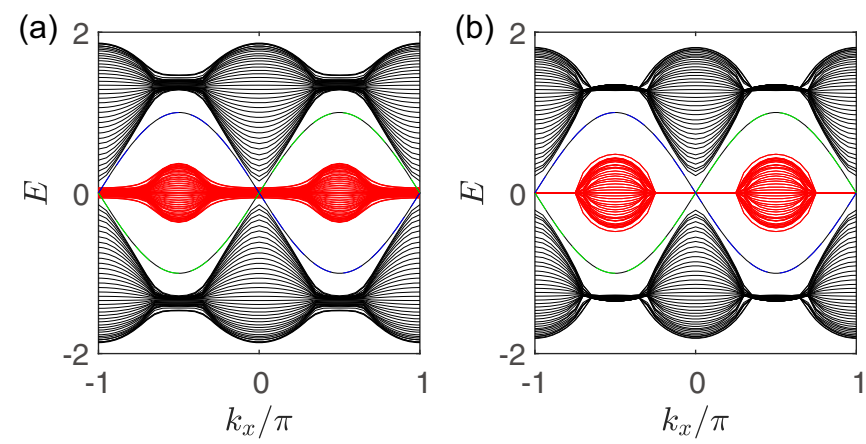

FIG. 11. Energy spectra under perturbation for the nonHermitian Chern insulator under OBC in the $y$ direction [Fig. 6(b)]. The real (imaginary) part of $E$ is in black (red). The dashed green and blue lines are the edge state energies $\pm t \sin k_{x}$ without perturbation. The inversion symmetric perturbations are (a) $V_{a_{j}}=V_{d_{j}}=-V_{b_{j}}=$ $-V_{c_{j}}=0.2 R_{j}$ and (b) $V_{a_{j}}=V_{d_{j}}=-V_{b_{j}}=-V_{c_{j}}=0.2 i R_{j}$, where $R_{j}$ is a random real number within region $[0,1]$. Other system parameters are identical to those in Fig. $7(\mathrm{e}), m=0, t=1$, and $\gamma=1 / 2$. The number of unit cells of the $1 \mathrm{D}$ projection lattice is twenty.

(detunings), as well as gain and loss, respectively. The inversion symmetry requires $V_{a_{j}}=V_{d_{j}}$ and $V_{b_{j}}=V_{c_{j}}$; the pseudoHermiticity holds under $V_{a_{j}}+V_{b_{j}}=0$ and $V_{c_{j}}+V_{d_{j}}=0$. The energy spectra of the lattice under perturbations are depicted in Fig. 11 for the comparison with the energy spectrum in the upper panel of Fig. 7(e). In Fig. 11(a), the perturbations are the inversion symmetric on-site potentials $V_{a_{j}}=V_{d_{j}}=$ $-V_{b_{j}}=-V_{c_{j}}=0.2 R_{j}$, where $R_{j}$ is a random real number within the region $[0,1]$ for each unit cell $j$. In Fig. 11(b), the perturbations are the inversion symmetric gain and loss $V_{a_{j}}=V_{d_{j}}=-V_{b_{j}}=-V_{c_{j}}=0.2 i R_{j}$. In both cases of Fig. 11, the in-gap edge state energies are unchanged and remain in the band gap, being robust to the inversion symmetric perturbations.
[1] M. Z. Hasan and C. L. Kane, Colloquium: Topological insulators, Rev. Mod. Phys. 82, 3045 (2010).

[2] D. Xiao, M.-C. Chang, and Q. Niu, Berry phase effects on electronic properties, Rev. Mod. Phys. 82, 1959 (2010).

[3] X.-L. Qi and S.-C. Zhang, Topological insulators and superconductors, Rev. Mod. Phys. 83, 1057 (2011).

[4] C.-K. Chiu, J. C. Y. Teo, A. P. Schnyder, and S. Ryu, Classification of topological quantum matter with symmetries, Rev. Mod. Phys. 88, 035005 (2016).

[5] A. Bansil, H. Lin, and T. Das, Colloquium: Topological band theory, Rev. Mod. Phys. 88, 021004 (2016).

[6] X.-G. Wen, Colloquium: Zoo of quantum-topological phases of matter, Rev. Mod. Phys. 89, 041004 (2017).

[7] N. P. Armitage, E. J. Mele, and A. Vishwanath, Weyl and Dirac semimetals in three-dimensional solids, Rev. Mod. Phys. 90, 015001 (2018).

[8] C. M. Bender, Making sense of non-Hermitian Hamiltonians, Rep. Prog. Phys. 70, 947 (2007).
[9] N. Moiseyev, Non-Hermitian Quantum Mechanics (University, Cambridge, England, 2011).

[10] I. Rotter and J. P. Bird, A review of progress in the physics of open quantum systems: theory and experiment, Rep. Prog. Phys. 78, 114001 (2015).

[11] V. V. Konotop, J. Yang, and D. A. Zezyulin, Nonlinear waves in $\mathscr{P} \mathscr{T}$-symmetric systems, Rev. Mod. Phys. 88, 035002 (2016).

[12] S. V. Suchkov, A. A. Sukhorukov, J. Huang, S. V. Dmitriev, C. Lee, and Y. S. Kivshar, Nonlinear switching and solitons in PT-symmetric photonic systems, Laser Photonics Rev. 10, 177213 (2016).

[13] L. Feng, R. El-Ganainy, and L. Ge, Non-Hermitian photonics based on parity-time symmetry, Nat. Photonics 11, 752 (2017).

[14] R. El-Ganainy, K. G. Makris, M. Khajavikhan, Z. H. Musslimani, S. Rotter, and D. N. Christodoulides, NonHermitian physics and $\mathcal{P} \mathcal{T}$ symmetry, Nat. Phys. 14, 11 (2018). 
[15] B.-Y. Xie, H.-F. Wang, X.-Y. Zhu, M.-H. Lu, Z. D. Wang, and Y.-F. Chen, Photonics meets topology, Opt. Exp. 26, 24531 (2018); S. K. Gupta, Y. Zou, X.-Y. Zhu, M.-H. Lu, L. Zhang, X.-P. Liu, and Y.-F. Chen, Parity-time symmetry in non-hermitian complex media, arXiv:1803.00794.

[16] D. Christodoulides and J. Yang, Parity-time Symmetry and Its Applications (Springer, 2018).

[17] M. S. Rudner and L. S. Levitov, Topological Transition in a Non-Hermitian Quantum Walk, Phys. Rev. Lett. 102, 065703 (2009).

[18] A. Szameit, M. C. Rechtsman, O. Bahat-Treidel, and M. Segev, $\mathcal{P} \mathcal{T}$-symmetry in honeycomb photonic lattices, Phys. Rev. A 84, 021806(R) (2011).

[19] K. Esaki, M. Sato, K. Hasebe, and M. Kohmoto, Edge states and topological phases in non-Hermitian systems, Phys. Rev. B 84, 205128 (2011).

[20] S. Diehl, E. Rico, M. A. Baranov, and P. Zoller, Topology by dissipation in atomic quantum wires, Nat. Phys. 7, 971 (2011); C.-E. Bardyn, M. A. Baranov, E. Rico, A. Imamoğlu, P. Zoller, and S. Diehl, Majorana Modes in Driven-Dissipative Atomic Superfluids with a Zero Chern Number, Phys. Rev. Lett. 109, 130402 (2012).

[21] Y. C. Hu and T. L. Hughes, Absence of topological insulator phases in non-Hermitian $\mathcal{P} \mathcal{T}$-symmetric Hamiltonians, Phys. Rev. B 84, 153101 (2011).

[22] G. Q. Liang and Y. D. Chong, Optical Resonator Analog of a Two-Dimensional Topological Insulator, Phys. Rev. Lett. 110, 203904 (2013).

[23] J. M. Zeuner, M. C. Rechtsman, Y. Plotnik, Y. Lumer, S. Nolte, M. S. Rudner, M. Segev, and A. Szameit, Observation of a Topological Transition in the Bulk of a Non-Hermitian System, Phys. Rev. Lett. 115, 040402 (2015).

[24] S. Malzard, C. Poli, and H. Schomerus, Topologically Protected Defect States in Open Photonic Systems with NonHermitian Charge-Conjugation and Parity-Time-Symmetry, Phys. Rev. Lett. 115, 200402 (2015).

[25] M. S. Rudner, M. Levin, and L. S. Levitov, Survival, decay, and topological protection in non-Hermitian quantum transport, arXiv:1605.07652.

[26] J. Hou, Z. Li, X.-W. Luo, Q. Gu, and C. Zhang, Topological bands and triply-degenerate points in non-Hermitian hyperbolic metamaterials, arXiv:1808.06972.

[27] W. Zhu, X. Fang, D. Li, Y. Sun, Y. Li, Y. Jing, and H. Chen, Simultaneous Observation of a Topological Edge State and Exceptional Point in an Open and Non-Hermitian Acoustic System, Phys. Rev. Lett. 121, 124501 (2018).

[28] Z. Oztas and C. Yuce, Spontaneously broken particle-hole symmetry in photonic graphene with gain and loss, Phys. Rev. A 98, 042104 (2018).

[29] P. Wang, S. Lin, G. Zhang, and Z. Song, Topological gapless phase in Kitaev model on square lattice, Sci. Rep. 7, 17179 (2017).

[30] Z.-Z. Li, X.-S. Li, L.-L. Zhang, and W.-J. Gong, $\mathcal{P} \mathcal{T}$ symmetry of the Su-Schrie er-Heeger model with imaginary boundary potentials and next-nearest-neighboring coupling, arXiv:1901.10688.

[31] X. Z. Zhang and Z. Song, Partial topological Zak phase and dynamical confinement in a non-Hermitian bipartite system, Phys. Rev. A 99, 012113 (2019).
[32] Y. V. Kartashov, V. V. Konotop, and L. Torner, Topological States in Partially- $\mathcal{P} \mathcal{T}$-Symmetric Azimuthal Potentials, Phys. Rev. Lett. 115, 193902 (2015).

[33] C. He, X.-C. Sun, X.-P. Liu, M.-H. Lu, Y. Chen, L. Feng, and Y.-F. Chen, Photonic topological insulator with broken timereversal symmetry, Proc. Natl. Acad. Sci. U. S. A. 113, 4924 (2016).

[34] F. K. Kunst, G. van Miert, and E. J. Bergholtz, Extended Bloch theorem for topological lattice models with open boundaries, Phys. Rev. B 99, 085427 (2019).

[35] W. B. Rui, Y. X. Zhao, and A. P. Schnyder, Topology and exceptional points of massive Dirac models with generic nonHermitian perturbations, Phys. Rev. B 99, 241110(R) (2019).

[36] L. Xiao, X. Zhan, Z. H. Bian, K. K. Wang, X. Zhang, X. P. Wang, J. Li, K. Mochizuki, D. Kim, N. Kawakami, W. Yi, H. Obuse, B. C. Sanders, and P. Xue, Observation of topological edge states in parity-time-symmetric quantum walks, Nat. Phys. 13, 1117 (2017).

[37] T. Rakovszky, J. K. Asbóth, and A. Alberti, Detecting topological invariants in chiral symmetric insulators via losses, Phys. Rev. B 95, 201407(R) (2017).

[38] C. Yin, H. Jiang, L. Li, R. Lü, and S. Chen, Geometrical meaning of winding number and its characterization of topological phases in one-dimensional chiral nonHermitian systems, Phys. Rev. A 97, 052115 (2018).

[39] H. Jiang, C. Yang, and S. Chen, Topological invariants and phase diagrams for one-dimensional two-band non-Hermitian systems without chiral symmetry, Phys. Rev. A 98, 052116 (2018).

[40] Y. Wang, L.-J. Lang, C. H. Lee, B. Zhang, and Y. D. Chong, Topologically enhanced harmonic generation in a nonlinear transmission line metamaterial, Nat. Commun. 10, 1102 (2019).

[41] S. Lin and Z. Song, Wide-range-tunable Diraccone band structure in a chiral-time-symmetric nonHermitian system, Phys. Rev. A 96, 052121 (2017).

[42] K. L. Zhang, P. Wang, and Z. Song, Majorana flat band edge modes of topological gapless phase in 2D Kitaev square lattice, Sci. Rep. 9, 4978 (2019).

[43] E. J. Bergholtz and J. C. Budich, Non-hermitian weyl physics in topological insulator ferromagnet junctions, Phys. Rev. Res. 1, 012003(R) (2019).

[44] K. Yamamoto, M. Nakagawa, K. Adachi, K. Takasan, M. Ueda, and N. Kawakami, Theory of Non-Hermitian Fermionic Superfluidity with a Complex-Valued Interaction, Phys. Rev. Lett. 123, 123601 (2019).

[45] H. Schomerus, Topologically protected midgap states in complex photonic lattices, Opt. Lett. 38, 1912 (2013).

[46] L. Jin, P. Wang, and Z. Song, Su-Schrieffer-Heeger chain with one pair of $\mathcal{P} \mathcal{T}$-symmetric defects, Sci. Rep. 7, 5903 (2017).

[47] C. Yuce, Edge states at the interface of non-Hermitian systems, Phys. Rev. A 97, 042118 (2018).

[48] L. J. Lang, Y. Wang, H. Wang, and Y. D. Chong, Effects of non-Hermiticity on Su-Schrieffer-Heeger defect states, Phys. Rev. B 98, 094307 (2018).

[49] S. Lieu, Topological phases in the non-Hermitian SuSchrieffer-Heeger model, Phys. Rev. B 97, 045106 (2018).

[50] A. K. Harter, T. E. Lee, and Y. N. Joglekar, $\mathscr{P} \mathscr{T}$-breaking threshold in spatially asymmetric Aubry-André and Harper 
models: Hidden symmetry and topological states, Phys. Rev. A 93, 062101 (2016).

[51] H. Jiang, L.-J. Lang, C. Yang, S.-L. Zhu, and S. Chen, Interplay of non-Hermitian skin effects and Anderson localization in non-reciprocal quasiperiodic lattices, Phys. Rev. B 100, 054301 (2019).

[52] C. Yuce, $\mathcal{P} \mathcal{T}$-symmetric Aubry-Andre model, Phys. Lett. A 378, 2024 (2014); Topological phase in a non-Hermitian $\mathcal{P} \mathcal{T}$ symmetric system, 379, 1213 (2015).

[53] Q.-B. Zeng, Y.-B. Yang, and Y. Xu, Topological phases in nonHermitian Aubry-André-Harper models, arXiv:1901.08060.

[54] S. Longhi, Topological Phase Transition in Non-Hermitian Quasicrystals, Phys. Rev. Lett. 122, 237601 (2019).

[55] L. Pilozzi and C. Conti, Topological lasing in resonant photonic structures, Phys. Rev. B 93, 195317 (2016).

[56] R. Wang, X. Z. Zhang, and Z. Song, Dynamical topological invariant for the non-Hermitian Rice-Mele model, Phys. Rev. A 98, 042120 (2018).

[57] F. K. Kunst, E. Edvardsson, J. C. Budich, and E. J. Bergholtz, Biorthogonal Bulk-Boundary Correspondence in Non-Hermitian Systems, Phys. Rev. Lett. 121, 026808 (2018).

[58] T. M. Philip, M. R. Hirsbrunner, and M. J. Gilbert, Loss of Hall conductivity quantization in a non-Hermitian quantum anomalous Hall insulator, Phys. Rev. B 98, 155430 (2018).

[59] Y. Chen and H. Zhai, Hall conductance of a non-Hermitian Chern insulator, Phys. Rev. B 98, 245130 (2018).

[60] K. Kawabata, K. Shiozaki, and M. Ueda, Anomalous helical edge states in a non-Hermitian Chern insulator, Phys. Rev. B 98, 165148 (2018).

[61] S. Yao, F. Song, and Z. Wang, Non-Hermitian Chern Bands, Phys. Rev. Lett. 121, 136802 (2018).

[62] M. Ezawa, Electric circuits for non-Hermitian Chern insulators, Phys. Rev. B 100, 081401(R) (2019).

[63] Y. Xu, S.-T. Wang, and L.-M. Duan, Weyl Exceptional Rings in a Three-Dimensional Dissipative Cold Atomic Gas, Phys. Rev. Lett. 118, 045701 (2017).

[64] A. Cerjan, M. Xiao, L. Yuan, and S. Fan, Effects of nonHermitian perturbations on Weyl Hamiltonians with arbitrary topological charges, Phys. Rev. B 97, 075128 (2018).

[65] C. Poli, M. Bellec, U. Kuhl, F. Mortessagne, and H. Schomerus, Selective enhancement of topologically induced interface states in a dielectric resonator chain, Nat. Commun. 6, 6710 (2015).

[66] S. Weimann, M. Kremer, Y. Plotnik, Y. Lumer, S. Nolte, K. G. Makris, M. Segev, M. C. Rechtsman, and A. Szameit, Topologically protected bound states in photonic, parity-timesymmetric crystals, Nat. Mater. 16, 433 (2017).

[67] M. Pan, H. Zhao, P. Miao, S. Longhi, and L. Feng, Photonic zero mode in a non-Hermitian photonic lattice, Nat. Commun. 9, 1308 (2018).

[68] P. St-Jean, V. Goblot, E. Galopin, A. Lemaître, T. Ozawa, L. Le Gratiet, I. Sagnes, J. Bloch, and A. Amo, Lasing in topological edge states of a one-dimensional lattice, Nat. Photonics 11, 651 (2017).

[69] M. Parto, S. Wittek, H. Hodaei, G. Harari, M. A. Bandres, J. Ren, M. C. Rechtsman, M. Segev, D. N. Christodoulides, and M. Khajavikhan, Edge-Mode Lasing in 1D Topological Active Arrays, Phys. Rev. Lett. 120, 113901 (2018).

[70] H. Zhao, P. Miao, M. H. Teimourpour, S. Malzard, R. El-Ganainy, H. Schomerus, and L. Feng, Topological hybrid silicon microlasers, Nat. Commun. 9, 981 (2018).

[71] S. Longhi, Non-hermitian gauged topological laser arrays, Ann. Phys. (Berlin) 530, 1800023 (2018).

[72] G. Harari, M. A. Bandres, Y. Lumer, M. C. Rechtsman, Y. D. Chong, M. Khajavikhan, D. N. Christodoulides, and M. Segev, Topological insulator laser: Theory, Science 359, eaar4003 (2018).

[73] M. A. Bandres, S. Wittek, G. Harari, M. Parto, J. Ren, M. Segev, D. Christodoulides, and M. Khajavikhan, Topological insulator laser: Experiments, Science 359, eaar4005 (2018).

[74] Y. V. Kartashov and D. V. Skryabin, Two-Dimensional Topological Polariton Laser, Phys. Rev. Lett. 122, 083902 (2019).

[75] M. Seclì, M. Capone, and I. Carusotto, Theory of chiral edge state lasing in a two-dimensional topological system, arXiv:1901.01290.

[76] L. Jin, Topological phases and edge states in a non-Hermitian trimerized optical lattice, Phys. Rev. A 96, 032103 (2017).

[77] H. Menke and M. M. Hirschmann, Topological quantum wires with balanced gain and loss, Phys. Rev. B 95, 174506 (2017).

[78] X. Ni, D. Smirnova, A. Poddubny, D. Leykam, Y. Chong, and A. B. Khanikaev, $\mathcal{P} \mathcal{T}$ phase transitions of edge states at $\mathcal{P} \mathcal{T}$ symmetric interfaces in non-Hermitian topological insulators, Phys. Rev. B 98, 165129 (2018).

[79] A. Ghatak and T. Das, Theory of superconductivity with nonHermitian and parity-time reversal symmetric Cooper pairing symmetry, Phys. Rev. B 97, 014512 (2018).

[80] K. Kawabata, Y. Ashida, H. Katsura, and M. Ueda, Paritytime-symmetric topological superconductor, Phys. Rev. B 98, 085116 (2018).

[81] K. Takata and M. Notomi, Photonic Topological Insulating Phase Induced Solely by Gain and Loss, Phys. Rev. Lett. 121, 213902 (2018).

[82] A. Yoshida, Y. Otaki, R. Otaki, and T. Fukui, Edge states, corner states, and flat bands in a two-dimensional $\mathscr{P} \mathscr{T}$-symmetric system, Phys. Rev. B 100, 125125 (2019).

[83] J. Hou, Z. Li, Q. Gu, and C. Zhang, Non-hermitian photonics based on charge-parity symmetry, arXiv:1904.05260.

[84] E. Cancellieri and H. Schomerus, PC-symmetry-protected edge states in interacting driven-dissipative bosonic systems, Phys. Rev. A 99, 033801 (2019).

[85] D. S. Borgnia, A. J. Kruchkov, and R.-J. Slager, Nonhermitian boundary modes, arXiv:1902.07217.

[86] S.-D. Liang and G.-Y. Huang, Topological invariance and global Berry phase in non-Hermitian systems, Phys. Rev. A 87, 012118 (2013).

[87] A. Ghatak and T. Das, New topological invariants in nonHermitian systems, J. Phys.: Condens. Matter 31, 263001 (2019).

[88] T. Ohashi, S. Kobayashi, and Y. Kawaguchi, Generalized Berry phase for a bosonic Bogoliubov system with exceptional points, arXiv:1904.08724.

[89] D. Leykam, K. Y. Bliokh, C. Huang, Y. D. Chong, and F. Nori, Edge Modes, Degeneracies, and Topological Numbers in NonHermitian Systems, Phys. Rev. Lett. 118, 040401 (2017).

[90] S. Lin, L. Jin, and Z. Song, Symmetry protected topological phases characterized by isolated exceptional points, Phys. Rev. B 99, 165148 (2019).

[91] F. Song, S. Yao, and Z. Wang, Non-hermitian topological invariants in real space, arXiv:1905.02211. 
[92] H. Shen, B. Zhen, and L. Fu, Topological Band Theory for Non-Hermitian Hamiltonians, Phys. Rev. Lett. 120, 146402 (2018).

[93] M. Papaj, H. Isobe, and L. Fu, Nodal arc of disordered Dirac fermions and non-Hermitian band theory, Phys. Rev. B 99, 201107(R) (2019).

[94] K. Yokomizo and S. Murakami, Non-Bloch Band Theory of Non-Hermitian Systems, Phys. Rev. Lett. 123, 066404 (2019).

[95] Z.-Y. Ge, Y.-R. Zhang, T. Liu, S.-W. Li, H. Fan, and F. Nori, Topological band theory for non-Hermitian systems from the Dirac equation, Phys. Rev. B 100, 054105 (2019).

[96] R. Wang, C. Li, X. Z. Zhang, and Z. Song, Dynamical bulkedge correspondence for degeneracy lines in parameter space, Phys. Rev. B 98, 014303 (2018).

[97] W. Hu, H. Wang, P. P. Shum, and Y. D. Chong, Exceptional points in a non-Hermitian topological pump, Phys. Rev. B 95, 184306 (2017).

[98] C. Yuce, Spontaneous topological pumping in non-Hermitian systems, Phys. Rev. A 99, 032109 (2019).

[99] Z. Gong, Y. Ashida, K. Kawabata, K. Takasan, S. Higashikawa, and M. Ueda, Topological Phases of NonHermitian Systems, Phys. Rev. X 8, 031079 (2018).

[100] K. Kawabata, S. Higashikawa, Z. Gong, Y. Ashida, and M. Ueda, Topological unification of time-reversal and particlehole symmetries in non-Hermitian physics, Nat. Commun. 10, 297 (2019).

[101] K. Kawabata, K. Shiozaki, M. Ueda, and M. Sato, Symmetry and topology in non-hermitian physics, arXiv:1812.09133.

[102] H. Zhou and J. Y. Lee, Periodic table for topological bands with non-Hermitian Bernard-LeClair symmetries, Phys. Rev. B 99, 235112 (2019).

[103] C.-H. Liu, H. Jiang, and S. Chen, Topological classification of non-Hermitian systems with reflection symmetry, Phys. Rev. B 99, 125103 (2019).

[104] K. Kawabata, T. Bessho, and M. Sato, Non-Hermitian Topology of Exceptional Points, Phys. Rev. Lett. 123, 066405 (2019).

[105] L. Li, C. H. Lee, and J. Gong, Geometric classification of nonHermitian topological systems through the singularity ring, Phys. Rev. B 100, 075403 (2019).

[106] T. Liu, Y.-R. Zhang, Q. Ai, Z. Gong, K. Kawabata, M. Ueda, and F. Nori, Second-Order Topological Phases in NonHermitian Systems, Phys. Rev. Lett. 122, 076801 (2019).

[107] C. H. Lee, L. Li, and J. Gong, Hybrid Higher-Order SkinTopological Modes in Non-Reciprocal Systems, Phys. Rev. Lett. 123, 016805 (2019).

[108] E. Edvardsson, F. K. Kunst, and E. J. Bergholtz, NonHermitian extensions of higher-order topological phases and their biorthogonal bulk-boundary correspondence, Phys. Rev. B 99, 081302(R) (2019).

[109] M. Ezawa, Higher-order topological electric circuits and topological corner resonance on the breathing kagome and pyrochlore lattices, Phys. Rev. B 98, 201402(R) (2018); NonHermitian boundary and interface states in nonreciprocal higher-order topological metals and electrical circuits, 99, 121411(R) (2019); Non-Hermitian higher-order topological states in nonreciprocal and reciprocal systems with their electric-circuit realization, 99, 201411(R) (2019).
[110] Z. Zhang, M. R. López, Y. Cheng, X. Liu, and J. Christensen, Non-Hermitian Sonic Second-Order Topological Insulator, Phys. Rev. Lett. 122, 195501 (2019).

[111] X.-W. Luo and C. Zhang, Higher-Order Topological Corner States Induced by Gain and Loss, Phys. Rev. Lett. 123, 073601 (2019)

[112] K. Moors, A. A. Zyuzin, A. Y. Zyuzin, R. P. Tiwari, and T. L. Schmidt, Disorder-driven exceptional lines and Fermi ribbons in tilted nodal-line semimetals, Phys. Rev. B 99, 041116(R) (2019)

[113] J. González and R. A. Molina, Topological protection from exceptional points in Weyl and nodal-line semimetals, Phys. Rev. B 96, 045437 (2017).

[114] J. Carlström, M. Stålmhammar, J. C. Budich, and E. J. Bergholtz, Knotted non-hermitian metals, Phys. Rev. B 99, 161115(R) (2019).

[115] C. H. Lee, G. Li, Y. Liu, T. Tai, R. Thomale, and X. Zhang, Tidal surface states as fingerprints of non-Hermitian nodal knot metals, arXiv:1812.02011.

[116] A. A. Zyuzin and A. Yu. Zyuzin, Flat band in disorder-driven non-Hermitian Weyl semimetals, Phys. Rev. B 97, 041203(R) (2018).

[117] R. A. Molina and J. González, Surface and 3D Quantum Hall Effects from Engineering of Exceptional Points in Nodal-Line Semimetals, Phys. Rev. Lett. 120, 146601 (2018).

[118] R. Okugawa and T. Yokoyama, Topological exceptional surfaces in non-Hermitian systems with parity-time and parityparticle-hole symmetries, Phys. Rev. B 99, 041202(R) (2019).

[119] J. C. Budich, J. Carlström, F. K. Kunst, and E. J. Bergholtz, Symmetry-protected nodal phases in non-Hermitian systems, Phys. Rev. B 99, 041406(R) (2019).

[120] T. Yoshida, R. Peters, N. Kawakami, and Y. Hatsugai, Symmetry-protected exceptional rings in two-dimensional correlated systems with chiral symmetry, Phys. Rev. B 99, 121101(R) (2019).

[121] Y.-J. Wu and J. Hou, Symmetry-protected localized states at defects in non-Hermitian systems, Phys. Rev. A 99, 062107 (2019).

[122] V. Kozii and L. Fu, Non-Hermitian topological theory of finitelifetime quasiparticles: prediction of bulk Fermi arc due to exceptional point, arXiv:1708.05841.

[123] T. Yoshida, R. Peters, and N. Kawakami, Non-Hermitian perspective of the band structure in heavy-fermion systems, Phys. Rev. B 98, 035141 (2018).

[124] H. Zhou, C. Peng, Y. Yoon, C. W. Hsu, K. A. Nelson, L. Fu, J. D. Joannopoulos, M. Soljačić, and B. Zhen, Observation of bulk Fermi arc and polarization half charge from paired exceptional points, Science 359, 1009 (2018).

[125] S. Malzard and H. Schomerus, Bulk and edge-state arcs in non-Hermitian coupled-resonator arrays, Phys. Rev. A 98, 033807 (2018).

[126] B. Zhen, C. W. Hsu, Y. Igarashi, L. Lu, I. Kaminer, A. Pick, S.-L. Chua, J. D. Joannopoulos, and M. Soljačić, Spawning rings of exceptional points out of Dirac cones, Nature (London) 525, 354 (2015).

[127] A. Cerjan, S. Huang, K. P. Chen, Y. Chong, and M. C. Rechtsman, Experimental realization of a Weyl exceptional ring, Nat. Photon. 13, 623 (2019). 
[128] H. Zhou, J. Y. Lee, S. Liu, and B. Zhen, Exceptional surfaces in $\mathcal{P} \mathcal{T}$-symmetric non-Hermitian photonic systems, Optica 6 , 190 (2019).

[129] T. E. Lee, Anomalous Edge State in a Non-Hermitian Lattice, Phys. Rev. Lett. 116, 133903 (2016).

[130] Y. Xiong, Why does bulk boundary correspondence fail in some non-hermitian topological models, J. Phys. Commun. 2, 035043 (2018).

[131] V. M. Martinez Alvarez, J. E. Barrios Vargas, and L. E. F. Foa Torres, Non-Hermitian robust edge states in one dimension: Anomalous localization and eigenspace condensation at exceptional points, Phys. Rev. B 97, 121401(R) (2018)

[132] V. M. Martinez Alvarez, J. E. Barrios Vargas, M. Berdakin, and L. E. F. Foa Torres, Topological states of nonHermitian systems, Eur. Phys. J. Special Topics 227, 1295 (2018).

[133] S. Yao and Z. Wang, Edge States and Topological Invariants of Non-Hermitian Systems, Phys. Rev. Lett. 121, 086803 (2018).

[134] L. Jin and Z. Song, Bulk-boundary correspondence in a nonHermitian system in one dimension with chiral inversion symmetry, Phys. Rev. B 99, 081103(R) (2019).

[135] P. Wang, L. Jin, and Z. Song, Non-Hermitian phase transition and eigenstate localization induced by asymmetric coupling, Phys. Rev. A 99, 062112 (2019).

[136] H. Wang, J. Ruan, and H. Zhang, Non-Hermitian nodalline semimetals with an anomalous bulk-boundary correspondence, Phys. Rev. B 99, 075130 (2019).

[137] C. H. Lee and R. Thomale, Anatomy of skin modes and topology in non-Hermitian systems, Phys. Rev. B 99, 201103(R) (2019).

[138] K. Luo, J. Feng, Y. X. Zhao, and R. Yu, Nodal manifolds bounded by exceptional points on non-hermitian honeycomb lattices and electrical-circuit realizations, arXiv:1810.09231.

[139] Z. Yang and J. Hu, Non-Hermitian Hopf-link exceptional line semimetals, Phys. Rev. B 99, 081102(R) (2019).

[140] L. Herviou, J. H. Bardarson, and N. Regnault, Defining a bulk-edge correspondence for non-Hermitian Hamiltonians via singular-value decomposition, Phys. Rev. A 99, 052118 (2019).

[141] T.-S. Deng and W. Yi, Non-Bloch topological invariants in a non-Hermtian domain-wall system, Phys. Rev. B 100, 035102 (2019).

[142] F. Song, S. Yao, and Z. Wang, Non-Hermitian skin effect and chiral damping in open quantum systems, arXiv:1904.08432.

[143] F. K. Kunst and V. Dwivedi, Non-Hermitian systems and topology: A transfer matrix perspective, Phys. Rev. B 99, 245116 (2019).

[144] H.-G. Zirnstein, G. Refael, and B. Rosenow, Bulk-boundary correspondence for non-Hermitian Hamiltonians via Green functions, arXiv:1901.11241.

[145] M. G. Silveirinha, Topological theory of non-Hermitian photonic systems, Phys. Rev. B 99, 125155 (2019).

[146] J.-Q. Cai, Q.-Y. Yang, Z.-Y. Xue, M. Gong, G.-C. Guo, and $\mathrm{Y}$. Hu, Interplay between non-Hermiticity and non-Abelian gauge potential in topological photonics, arXiv:1812.02610.

[147] K. L. Zhang, H. C. Wu, L. Jin, and Z. Song, Topological phase transition independent of system non-hermiticity, Phys. Rev. B 100, 045141 (2019).
[148] X. M. Yang, P. Wang, L. Jin, and Z. Song, Visualizing topology of real-energy gapless phase arising from exceptional point, arXiv:1905.07109.

[149] M. Creutz, End States, Ladder Compounds, and Domain-Wall Fermions, Phys. Rev. Lett. 83, 2636 (1999).

[150] D.-W. Zhang, Y.-Q. Zhu, Y. X. Zhao, H. Yan, and S.-L. Zhu, Topological quantum matter with cold atoms, Adv. Phys. 67, 253 (2019)

[151] W. D. Heiss and H. L. Harney, The chirality of exceptional points, Eur. Phys. J. D 17, 149 (2001); W. D. Heiss, The physics of exceptional points, J. Phys. A: Math. Theor. 45, 444016 (2012).

[152] C. Dembowski, H.-D. Gräf, H. L. Harney, A. Heine, W. D. Heiss, H. Rehfeld, and A. Richter, Experimental Observation of the Topological Structure of Exceptional Points, Phys. Rev. Lett. 86, 787 (2001); C. Dembowski, B. Dietz, H.-D. Gräf, H. L. Harney, A. Heine, W. D. Heiss, and A. Richter, Encircling an exceptional point, Phys. Rev. E 69, 056216 (2004).

[153] M. V. Berry, Physics of non-Hermitian degeneracies, Czech. J. Phys. 54, 1039 (2004); A. A. Mailybaev, O. N. Kirillov, and A. P. Seyranian, Geometric phase around exceptional points, Phys. Rev. A 72, 014104 (2005).

[154] R. Uzdin, A. Mailybaev, and N. Moiseyev, On the observability and asymmetry of adiabatic state flips generated by exceptional points, J. Phys. A 44, 435302 (2011).

[155] J. Doppler, A. A. Mailybaev, J. Böhm, U. Kuhl, A. Girschik, F. Libisch, T. J. Milburn, P. Rabl, N. Moiseyev, and S. Rotter, Dynamically encircling an exceptional point for asymmetric mode switching, Nature (London) 537, 76 (2016).

[156] H. Xu, D. Mason, L. Jiang, and J. G. E. Harris, Topological energy transfer in an optomechanical system with exceptional points, Nature (London) 537, 80 (2016).

[157] K. Ding, G. Ma, M. Xiao, Z. Q. Zhang, and C. T. Chan, Emergence, Coalescence, and Topological Properties of Multiple Exceptional Points and their Experimental Realization, Phys. Rev. X 6, 021007 (2016).

[158] X.-L. Zhang, S. Wang, B. Hou, and C. T. Chan, Dynamically Encircling Exceptional Points: In situ Control of Encircling Loops and the Role of the Starting Point, Phys. Rev. X 8, 021066 (2018).

[159] J. Wiersig, Enhancing the Sensitivity of Frequency and Energy Splitting Detection by Using Exceptional Points: Application to Microcavity Sensors for SingleParticle Detection, Phys Rev. Lett. 112, 203901 (2014).

[160] Z.-P. Liu, J. Zhang, S. K. Özdemir, B. Peng, H. Jing, X.-Y. Lü, C.-W. Li, L. Yang, F. Nori, and Y. X. Liu, Metrology with $\mathcal{P} \mathcal{T}$ Symmetric Cavities: Enhanced Sensitivity near the $\mathcal{P} \mathcal{T}$-Phase Transition, Phys. Rev. Lett. 117, 110802 (2016).

[161] W. Chen, S. K. Özdemir, G. Zhao, J. Wiersig, and L. Yang, Exceptional points enhance sensing in an optical microcavity, Nature (London) 548, 192 (2017).

[162] H. Hodaei, A. U. Hassan, S. Wittek, H. Garcia-Gracia, R. El-Ganainy, D. N. Christodoulides, and M. Khajavikhan, Enhanced sensitivity at higher-order exceptional points, Nature (London) 548, 187 (2017).

[163] B. Midya, H. Zhao, and L. Feng, Non-Hermitian photonics promises exceptional topology of light, Nat. Commun. 9, 2674 (2018).

[164] M.-A. Miri and A. Alù, Exceptional points in optics and photonics, Science 363, 42 (2019). 
[165] L. Jin, H. C. Wu, B.-B. Wei, and Z. Song, Hybrid exceptional point created from type III Dirac point, arXiv:1908.10512.

[166] X. L. Qi, Y. S. Wu, and S. C. Zhang, Topological quantization of the spin hall effect in two-dimensional paramagnetic semiconductors, Phys. Rev. B 74, 085308 (2006).

[167] S.-L. Zhang and Q. Zhou, Two-leg Su-Schrieffer-Heeger chain with glide reflection symmetry, Phys. Rev. A 95, 061601(R) (2017).

[168] C. Li, S. Lin, G. Zhang, and Z. Song, Topological nodal points in two coupled Su-Schrieffer-Heeger chains, Phys. Rev. B 96, 125418 (2017).

[169] A. Mostafazadeh, Pseudo-Hermiticity versus $\mathcal{P} \mathcal{T}$ symmetry: The necessary condition for the reality of the spectrum of a non-Hermitian Hamiltonian, J. Math. Phys. 43, 205 (2002).

[170] L. Jin and Z. Song, Hermitian dynamics in a class of pseudoHermitian networks, Phys. Rev. A 84, 042116 (2011).

[171] T. Fukui, Y. Hatsugai, and H. Suzuki, Chern numbers in discretized Brillouin zone: efficient method of computing (spin) Hall conductances, J. Phys. Soc. Jap. 74, 1674 (2005).

[172] F. Liu and K. Wakabayashi, Novel Topological Phase with a Zero Berry Curvature, Phys. Rev. Lett. 118, 076803 (2017).

[173] R. Resta, Macroscopic polarization in crystalline dielectrics: the geometric phase approach, Rev. Mod. Phys. 66, 899 (1994).

[174] S. Longhi, D. Gatti, and G. Della Valle, Robust light transport in non-Hermitian photonic lattices, Sci. Rep. 5, 13376 (2015); Non-Hermitian transparency and oneway transport in low- dimensional lattices by an imaginary gauge field, Phys. Rev. B 92, 094204 (2015).

[175] M. Aidelsburger, M. Atala, M. Lohse, J. T. Barreiro, B. Paredes, and I. Bloch, Realization of the Hofstadter Hamiltonian with Ultracold Atoms in Optical Lattices, Phys. Rev. Lett. 111, 185301 (2013); M. Aidelsburger, M. Lohse, C. Schweizer, M. Atala, J. T. Barreiro, S. Nascimbène, N. R. Cooper, I. Bloch, and N. Goldman, Measuring the Chern number of Hofstadter bands with ultracold bosonic atoms, Nat. Phys. 11, 162 (2015).

[176] N. Goldman, J. C. Budich, and P. Zoller, Topological quantum matter with ultracold gases in optical lattices, Nat. Phys. 12, 639 (2016).

[177] N. R. Cooper, J. Dalibard, and I. B. Spielman, Topological bands for ultracold atoms, Rev. Mod. Phys. 91, 015005 (2019).

[178] M. Hafezi, E. A. Demler, M. D. Lukin, and J. M. Taylor, Robust optical delay lines with topological protection, Nat. Phys. 7, 907 (2011); M. Hafezi, S. Mittal, J. Fan, A. Migdall, and J. M. Taylor, Imaging topological edge states in silicon photonics, Nat. Photonics 7, 1001 (2013).

[179] S. Mittal, J. Fan, S. Faez, A. Migdall, J. M. Taylor, and M. Hafezi, Topologically Robust Transport of Photons in a Synthetic Gauge Field, Phys. Rev. Lett. 113, 087403 (2014); S. Mittal, S. Ganeshan, J. Fan, A. Vaezi, and M. Hafezi, Measurement of topological invariants in a 2D photonic system, Nat. Photonics 10, 180 (2016); S. Mittal, E. A. Goldschmidt, and M. Hafezi, A topological source of quantum light, Nature (London) 561, 502 (2018). 